\title{
Size-Exclusion Simulated Moving Bed for Separating Organophosphorous Flame Retardants from a Polymer
}

Authors: George S. Weeden, Jr., Lei Ling, Nicholas H. Soepriatna, and Nien-Hwa Linda Wang*

Affiliations:

School of Chemical Engineering, Purdue University, West Lafayette, Indiana 47907-2100, United States

*Corresponding author. Phone: (765) 494-4081; fax: (765) 494-0805; e-mail:

wangn@ecn.purdue.edu. 
1 Abstract: Over 500,000 tonnes of flame retardants in electronic wastes are consigned to landfills

2 each year. A room-temperature, size-exclusion simulated moving bed (SEC-SMB) was

3 developed to recover high purity (>99\%) flame retardants with high yield (>99\%). The SSWD

4 method for ternary mixtures was developed for SEC-SMB. Fourteen decision variables were

5 optimized to obtain the lowest separation cost within one minute. The estimated cost is less than

$6 \quad 10 \%$ of the purchase cost of the flame retardants. The estimated cost of the optimized SEC-SMB

7 is less than $3 \%$ of that of a conventional batch SEC processes. Fast startup methods were

8 developed to reduce the SMB start-up time by more than 18 fold. SEC-SMB can be an

9 economical method for separating small molecules from polymers.

10

11 Keywords: Simulated Moving Bed; Size-Exclusion; Electronic Waste; Phosphorous Flame

12 Retardants; Polymer; Optimization 


\section{$13 \quad \mathbf{1 . 0}$ Introduction}

14 Over 50 million tonnes of waste electrical and electronic equipment (WEEE) are

15 generated worldwide each year and they are growing at a rate of 3-5\% per year [1-5]. Polymeric

16 materials make up about one third of that weight [6,7]. The United States Environmental

17 Protection Agency estimated that less than $20 \%$ of the wastes, mostly metals and glass, are

18 recycled $[8,9]$. The rest of the wastes are currently stored in landfills or scattered in the oceans

19 around the world $[10,11]$. Slow degradation of the polymers releases toxic chemicals, which

20 potentially endanger wildlife and affect our food supply [12,13]. Recovering polymers and

21 valuable chemicals from the wastes can reduce these environmental hazards and the amount of

22 raw materials and energy required for synthesis.

23 Recovery of high-purity polymers from a polymer waste is challenging because many

24 polymers have similar physical properties and broad, overlapping molecular weight (MW)

25 distributions. Single solvents do not have the selectivity to recover high-purity polymers with

26 high yield. A room-temperature, mixed-solvent process, Sequential Extraction for Polymer

27 Recovery (SEPoR), was developed to recover polymers from wastes [14,15]. The solvent

28 compositions are designed using a combination of Hansen Solubility Parameter (HSP) theory,

29 gradient polymer elution chromatography (GPEC), and solubility tests. SEPoR has already been

30 developed for recovering high purity (>99\%) polycarbonates (PCs) with high (>95\%) yield from

31 a computer waste. SEPoR uses $84 \%$ less energy than chemical synthesis and can reduce $\mathrm{CO}_{2}$

32 emissions and reduce raw materials from petroleum used to synthesize virgin polymers [15].

33 poly(acrylonitrile-co-butadiene-co-styrene) (ABS) is removed as a separate, solid product. Flame

34 retardants (FRs) and a polymer, poly(styrene-co-acrylonitrile) (SAN), are discharged in a side

35 stream of 50/50 (vol \%) acetone (ACE)/dichloromethane (DCM). Recovery of the FRs and SAN 
36 from the side stream is economically desirable and beneficial to the environment, and it is the

37 focus of this study.

Flame retardants, such as resorcinol bis-diphenylphosphate (RDP) and bisphenol A bis-

39 diphenylphosphate (BPADP), are added to polymers in order to inhibit the spread of flames in

40 case of fire $[16,17]$. Many flame retardants containing bromine or other halogens are being

41 preplaced by organophosphorous FRs, which are safer and more environmentally benign $[18,19]$.

42 This study focuses on the recovery of RDP (575-4,025 Da), BPADP (693 Da), and SAN

$43(50,000-150,000 \mathrm{Da})$ from one of the side streams of the SEPoR process for PC recovery, Fig. 1.

44 The molecular structures for the FRs and SAN are shown in Table 1.

45 The FRs are the most valuable components in the polymer wastes by weight. Polymers in

46 WEEE have 10 wt $\%$ or more FRs. More than 500,000 tonnes of FRs potentially could be

47 recovered annually from WEEE. Furthermore, FRs must be removed for recovering high-purity

48 polymers from wastes.

49 The existing literature on organophosphorous FRs in polymer wastes focuses on

50 analytical methods for detection [20-23]. Microwave-assisted extraction, combined with gel

51 permeation chromatography and mass spectrometry, was used to detect organophosphorous FRs

52 in biological samples from fish and birds [24]. Solid phase extraction, combined with reverse

53 phase chromatography, was used to detect FRs in water samples [25]. Pressurized liquid

54 extraction (acetonitrile and water), combined with gas chromatography, was used to analyze

55 sediment samples [26]. No literature has been found for recovering organophosphorous FRs

56 from polymer waste at large scale.

57 Since the MW of the FRs and SAN differ by two orders of magnitude, size-exclusion 58 chromatography (SEC) is a potential separation technique. SEC has been widely used for 
59 analyzing polymer mixtures [27,28]. SEC is a batch chromatography process, which is less

60 efficient than simulated moving-bed (SMB) chromatography for large-scale production. SMB

61 can achieve high product purity without sacrificing product yield. It also requires much less

62 solvent and can have an order of magnitude higher adsorbent productivity. For this reason, this

63 study focuses on developing an economical SMB process based on size exclusion principles

64 (SEC-SMB) for separating the FRs from SAN.

SEC-SMB is a continuous chromatography process. The efficiency of SMB comes from

66 a circular column configuration (a loop) and multiple inlet and outlet ports that divide this loop

67 into various sections (or zones) with different flow rates. Figure 2 illustrates a typical 4-zone

68 SMB with two columns per zone (2-2-2-2 configuration). A feed mixture is continuously input to

69 the loop, while two products are drawn from two separate product ports. The ports move

70 periodically along the loop to follow migrating solute bands. The time between port switches is

71 called the switching time, or step time. Separation is achieved by controlling the flowrate in each

72 zone such that the concentration waves of specific solutes are confined in specific zones. As seen

73 in Fig. 2, the slow component is never present in Zone IV while the fast component is never

74 present in Zone I. By confining the advancing and trailing concentration waves in their

75 respective zones, pure slow and fast products can be continuously removed in the respective

76 product ports. Only partial separation of the two solute bands in the loop is needed to recover

77 two high-purity products with high yields. As a result, a large fraction of the stationary phase in

78 the loop can be utilized and dilution of the bands can be reduced. For this reason, SMB can have

79 an order of magnitude higher sorbent productivity and lower solvent consumption than

80 conventional batch chromatography. SMB also requires fewer operators, takes up less floor

81 space, and uses less costly equipment for large-scale production. 
Although SMB has many advantages, it is not widely used for separation of complex

83 mixtures. Design of a four-zone SMB requires specification of four zone flowrates, four zone

84 lengths, and a step time. Dynamic concentration wave propagation in SMB is affected by

85 multiple inlet and outlet streams and is not well understood. SMB experiments are also costly

86 and time-consuming when developing a process.

87 The only large-scale SEC-SMB process is UOP's Molex ${ }^{\mathrm{TM}}$ process, which separates n-

88 paraffins from branched/cyclic hydrocarbons [29,30]. Lab-scale SEC-SMB studies have been

89 reported for several important compounds. Some of these systems include influenza [31] and

90 adenovirus [32] production for vaccines, recombinant protein purification [33], insulin

91 purification [34-37], lactose removal from human milk [38], and polyethylene glycol

92 fractionation by MW [39]. Only lab-scale operations have been performed for high MW

93 molecules $(\mathrm{MW}>5 \mathrm{kDa})$. No studies on the separation of organophosphorous FRs from large

94 polymers using SEC-SMB have been reported in the literature.

95 The five operating parameters (four zone velocities and one port velocity) can be

96 designed by a number of methods, including the local equilibrium theory or "triangle" theory,

97 which is widely used and works well for ideal systems (no mass transfer resistance) [40].

98 However, for non-ideal systems (with mass transfer resistance), this theory only gives the range

99 of possible operating parameters where separation of the components will occur. It does not

100 guarantee purity or yield and it does not give optimum operating parameters for non-ideal

101 systems.

102 Another method for SMB design is the Standing Wave Design (SWD), which was first

103 developed by Ma and Wang in 1997 for binary, linear adsorption systems with mass transfer

104 resistances [41]. For fixed yields, material properties (size-exclusion factors, diffusivities, 
105 particle porosity, bed void fraction, and particle size), and equipment parameters (column length,

106 dead volume, column configuration, and pressure limit), the SWD determines the five optimum

107 operating parameters to maximize productivity and minimize solvent consumption. It was

108 extended to multicomponent linear systems [42] and nonlinear systems [43-45]. Pressure limit

109 considerations were incorporated into the SWD [46] by checking that the resulting operating

110 parameters did not violate the pressure constraint.

111 In a recent study, the SWD equations for SEC-SMB separations of binary mixtures were

112 simplified using dimensionless groups. This new method was called the Speedy Standing Wave

113 Design (SSWD) because it can quickly provide overviews of solvent consumption, sorbent

114 productivity, and separation cost (with given cost functions) [47]. For given material properties,

115 yields, and column configuration, the SSWD can be used to determine the operating parameters

116 and column length from two key dimensionless groups: (1) the ratio of the step time to the

117 intraparticle diffusion time and (2) the ratio of the dispersion time to the step time.

118 The objectives of this study are to: (1) extend the SSWD from binary to ternary mixtures;

119 (2) estimate the material properties of a selected system (sorbent, solvent, solutes); (3) use the 120 extended SSWD to design the operating parameters of SEC-SMBs for recovering both FRs and 121 SAN with high purity and high yield; (4) experimentally test the design method and verify the 122 estimated material properties; (5) develop and test fast startup methods to reduce the startup time 123 of SEC-SMB; and (6) investigate the economic feasibility of the SEC-SMBs at large scale. 


\subsection{Theory}

125

129 velocity of a specific component in each zone. Those specific concentration waves are resistance), a difference between port velocity and concentration wave velocity is used to confine

132 the waves by focusing them toward the zone boundaries. This concept is illustrated for a specific

133 binary SEC system in Fig. 3a. Arrows in Fig. 3 indicate the confined wave in each zone. The

134 trailing wave of the slow-moving component (RDP) is focused and confined in Zone I and that 135 of the fast-moving component (SAN) is confined in Zone II. The advancing waves of RDP and 136 SAN are confined in Zones III and IV, respectively.

138 in Eq. (1).

$$
u_{w, 1}^{I I}=\frac{u_{0}^{I I}}{1+\phi \delta_{1}}=v+\Delta_{1}^{I I}
$$

$141 \quad u_{w, 2}^{I I I}=\frac{u_{0}^{I I I}}{1+\phi \delta_{2}}=v-\Delta_{2}^{I I I}$

$142 \quad u_{w, 1}^{I V}=\frac{u_{0}^{I V}}{1+\phi \delta_{1}}=v-\Delta_{1}^{I V}$

$143 u_{F}=u_{0}^{I I I}-u_{0}^{I I}$ 
144 where $u_{w, i}^{j}$ is the wave velocity of component $\mathrm{i}$ in zone $\mathrm{j} ; u_{0}^{j}$ is the interstitial velocity of the

145 fluid in zone $\mathrm{j}$ (zone velocity); $\phi$ is the phase ratio, $\phi=\left(1-\varepsilon_{b}\right) / \varepsilon_{b}$, with $\varepsilon_{b}$ designating the

146 interparticle void fraction; $\delta_{i}$ is the apparent retention factor for component $\mathrm{i} ; v$ is the port

147 velocity, which is equal to the column length $\left(L_{c}\right)$ divided by the step time $\left(t_{s}\right) ; \Delta_{i}^{j}$ is the mass

148 transfer correction term for non-ideal systems, Eq. (A.1); and $u_{F}$ is the feed velocity. If feed

149 velocity (flowrate) and mass transfer parameters are given, Eq. (1) can be used to determine the

150 port velocity and four zone velocities. Details are discussed in Appendix A.

\subsection{Speedy Standing Wave Design (SSWD)}

The SWD equations, Eq. (1), can be used to determine the operating parameters for given

153 yields, material properties, and equipment parameters. In a recent study, these equations were

154 simplified using dimensionless groups for SEC-SMB separation of a binary mixture. In this

155 study, the SSWD equations are extended to ternary mixtures. Because there are three

156 components and only two product ports, there are three splitting options (splits).

The first option is to recover the fastest solute (SAN) in the raffinate and to recover the

158 slower solutes (BPADP and RDP) in the extract, Fig. 3b. The desired splitting can be achieved

159 by confining the trailing wave of RDP in Zone I, the trailing wave of SAN in Zone II, the

160 advancing wave of BPADP in Zone III, and the advancing wave of SAN in Zone IV. The trailing

161 wave of BPADP is automatically confined ("pinched," indicated by an asterisk in Fig. 3b) in

162 Zone I since it moves faster than the trailing wave of RDP. Similarly, the advancing wave of

163 RDP is pinched in Zone III since it moves slower than the advancing wave of BPADP.

The second option is to recover the slowest solute (RDP) in the extract and to recover

165 SAN and BPADP in the raffinate, Fig. 3c. The desired splitting can be achieved by confining the

166 trailing wave of RDP in Zone I, the trailing wave of BPADP in Zone II, the advancing wave of 
167 RDP in Zone III, and the advancing wave of SAN in Zone IV. The trailing wave of SAN is 168 automatically confined ("pinched," indicated by an asterisk in Fig. 3c) in Zone II since it moves 169 faster than the trailing wave of BPADP. Similarly, the advancing wave of BPADP is pinched in 170 Zone IV since it moves slower than the advancing wave of SAN.

171 The third option is to allow one solute to be recovered in both product streams. The 172 operating parameters for this option can be determined using the SSWD for a binary mixture of 173 the two remaining components.

174 The key dimensionless groups used to simplify the SWD equations are shown in Table 2, 175 where $\alpha_{i}$ is the selectivity of component i relative to the fast solute and is equal to the ratio of the 176 apparent retention factor for component $\mathrm{i}$ over that of the fast solute; $\beta_{i}^{j}$ is the natural logarithm 177 of the ratio of the highest concentration to the lowest concentration of the standing wave of 178 component $\mathrm{i}$ in zone $\mathrm{j}$ (the value of $\beta_{i}^{j}$ is directly related to the yield of component $\mathrm{i}$ and the zone 179 velocities), Eq. (A.3); $N^{j}$ is the number of columns in zone $\mathrm{j}$; $\lambda_{i}$ is the ratio of the size-exclusion 180 factor $\left(K_{s e, i}\right)$ for component i over that of the fast solute (SAN); $\gamma_{i}$ is the ratio of the diffusivity of 181 component i $\left(D_{p, i}\right)$ over that of SAN; and $\Gamma^{j}$ is the ratio of the axial dispersion coefficient of zone $182 \mathrm{j}$ over that of Zone IV $\left(E_{b, i}^{j} / E_{b, 1}^{I V}\right)$.

183 The dimensionless group which accounts for mass transfer effects due to intraparticle 184 diffusion is $N_{D}^{*}$ and is defined in Eq. (2) for splitting the fast solute from the slower solutes,

$185 \quad N_{D}^{*}=\frac{\left(\alpha_{2}-1\right)}{\phi \delta_{1}} \frac{\phi \varepsilon_{p} K_{s e, 1} D_{p, 1}}{R_{p}{ }^{2}} \frac{L_{c}}{v}=\frac{\left(\alpha_{2}-1\right)}{\phi \delta_{1}} \frac{t_{s}}{t_{D, 1}}$

186 where $\varepsilon_{p}$ is the particle porosity; $R_{p}$ is the particle radius; and $t_{D, 1}$ is the characteristic diffusion 187 time of solute $1, t_{D, 1}=R_{p}{ }^{2} / D_{p, 1}$. A large $N_{D}^{*}$ means that the solutes can diffuse into and out of the 188 particles many times between port switches, which in turn means that the wave spreading due to 
189 diffusion is small. A small $N_{D}^{*}$ means that there is not enough time for the solute to diffuse

190 through the particle within the step time, which in turn means wave spreading due to diffusion is

191 significant.

192 The dimensionless group which accounts for mass transfer effects due to dispersion is the 193 modified Peclet number $\left(P_{e b}^{*}\right)$, which is defined in Eq. (3) for splitting the fast solute from two 194 slower solutes,

$195 P_{e b}^{*}=\phi\left(\delta_{2}-\delta_{1}\right) \frac{v L_{c}}{E_{b, 1}^{I V}}=\phi\left(\delta_{2}-\delta_{1}\right) \frac{t_{D a x}}{t_{s}}$

196 where $t_{\text {Dax }}$ is a characteristic dispersion time, which is equal to ${L_{c}}^{2}$ divided by $E_{b, 1}^{I V}$. A very large $197 P_{e b}^{*}$ indicates that the dispersion time is much greater than the step time, meaning there is little 198 effect of dispersion on the wave spreading. If $P_{e b}^{*}$ is small, then the dispersion time is closer to 199 the step time and the effects from dispersion are significant.

200 The detailed derivation of the SSWD equations for ternary mixtures is shown in 201 Appendix A. The resulting equations for two different ternary splits are shown in Table 3, Eq. 202 (4) and Eq. (5). Because Split 1 generates pure SAN in the raffinate and a mixture of FRs in the 203 extract, both products are valuable. The SAN product is only valuable if it is pure, whereas the 204 two FRs can be recycled as a mixture. The other splits do not produce two useful products. For 205 this reason, only Split 1 is tested experimentally and verified with simulations. The theoretical 206 results for the other splits are presented for completeness and can be used for other applications.

207 Pressure limitation can also be a major factor for some applications. For the separation of 208 FRs from SAN to be economical, a concentrated feed solution is needed to reduce the overall 209 separation cost, Eq. (B.1-B.4). A concentrated polymer solution can be very viscous, which in 210 turn can lead to a high pressure drop. Systems can also be limited by pressure drop per unit 211 packing length if the sorbent is very soft [34] or by the maximum pressure allowed by pumps, 
212 valves, or column casings. The pressure drop for laminar flow across a uniformly packed bed of

213 monodisperse, spherical particles can be estimated using the simplified Ergun equation, Eq. (6),

214 [48].

$215 \quad \frac{\Delta P}{L^{I}}=\frac{150 \mu u_{0}^{I} \phi^{2}}{4 R_{p}^{2}}$

216 The pressure drop across a zone is represented by $\Delta P$ and the viscosity of the fluid is represented

217 by $\mu$. Zone I always has the largest velocity and its value is limited by the maximum pressure

218 allowed. The viscosity of the mobile phase in Zone I is assumed to have the same viscosity as the

219 feed. Equation (4a) can be substituted into Eq. (6) and then rearranged to find a dimensionless

220 group which represents the pressure limitations of the system. This group was derived by

221 Weeden and Wang [47] and is defined for Splits 1 and 2 in Eq. (7) and Eq. (8), respectively. For

222 fixed pressure limits, column configuration $\left(N^{j}\right)$, and material properties $\left(\delta_{i}, R_{p}, \mu, \phi, \lambda_{i}, \gamma_{i}\right)$, the

223 values of $N_{D}^{*}$ and $P_{e b}^{*}$ which will satisfy the pressure limit are bounded by Eq. (7) or Eq. (8).

\subsection{Optimization of Decision Variables}

The 15 decision variables that can be optimized are column configuration (4), column

226 length, dead volume, three yields, particle size, and five operating parameters, Fig. 4. The SSWD

227 equations in Table 2 determine the zone velocities and port velocity for given material

228 properties, yields, and equipment parameters. Once the zone velocities are known, the solvent

229 consumption, sorbent productivity, and total cost can be easily calculated (for given cost

230 functions, Appendix B).

231 The dead volume has been shown to have little effect on SEC-SMB cost when it is less

232 than $2 \%$ of the column volume [47], so it was kept at the experimental value of $1.9 \%$. For the 233 separation of FRs from SAN, $D V$ is fixed and the remaining 14 are optimized, Section 4.7. The 
234 algorithm used to optimize the 14 decision variables (column configuration, column length,

235 yields, particle radius, zone velocities, and port velocity) to achieve minimum cost is shown in

236 Appendix C, Fig. C.1.

\subsection{Preloading Strategies for Fast Startup of SMB}

The SWD method gives the operating parameters to achieve desired product purity or

239 yield at steady state. However, it does not give the time for an SMB system to reach cyclic

240 steady state (startup time), which can be determined using experiments or a simulation program,

241 such as VERSE (description in Supplementary Materials, S.1). Generally, for an SMB starting

242 from clean columns to reach cyclic steady state, the ports must move around the loop three or

243 more times (cycles) [44]. Fast startup methods are needed to significantly reduce the time and

244 materials required for startup.

245 A number of strategies for startup of SMB systems have been reported in the literature.

246 One proposed method is for the operating parameters to be different from their cyclic steady-

247 state values [49,50]. Xie et al. [36] proposed the following preloading strategy to reduce SMB

248 startup time. Several columns were preloaded with feed solution. The columns were then

249 connected and elution was used to obtain approximately the steady-state concentration profiles

250 predicted by VERSE.

251 In this study, two strategies were developed and compared to the literature method of Xie

252 et al. and startup from clean columns. To better approximate the steady-state column profiles, the

253 first strategy involves preloading different columns with solutions of different concentrations,

254 which were determined by VERSE simulations. The columns in Zone I were preloaded with a

255 solution of the slow-moving solute at the steady-state concentration of the extract obtained from

256 VERSE. Similarly, the columns in Zone III were preloaded with a solution of the fast-moving 
257 solute at the steady-state concentration of the raffinate obtained from VERSE. The columns in

258 Zone II were preloaded with a solution of both solutes at the same concentrations as the other

259 two preloading solutions. The columns were then connected and elution was used to shift the

260 solute bands into their stead-state positions. The second strategy uses the same method as the

261 first, except the preloading concentrations were set to be the same as the product concentrations

262 determined by mass balance using the zone velocities determined from SSWD.

\section{3.0 Experimental}

\subsection{Materials}

The polymer and the flame retardants were dissolved in a 50/50 (vol \%) mixture of DCM

266 and ACE. The solvents were obtained from Mallinckrodt and were more than 99\% pure.

267 Isopropanol (>99\%) was used in column packing and was also purchased from Mallinckrodt.

268 Blue dextran (average molecular weight $=2,000,000 \mathrm{Da}$ ) was obtained from Sigma-Aldrich. The

269 mobile phase used for the HPLC analysis of the different components was a mixture of

270 acetonitrile (ACN, >99.5\% pure from Mallinckrodt) and tetrahydrofuran (THF, > 99.9\% pure

271 from Sigma-Aldrich) without stabilizer. The polymer, SAN, and the flame retardants, RDP and

272 BPADP, were provided by SABIC Innovative Plastics. The packing material used for the SMB

273 experiments was Amberlite XAD 1180N, which was purchased from DOW Water and Process

274 Solutions. The average particle size was $450 \mu \mathrm{m}$ with an average pore size of $450 \AA$.

\subsection{Equipment}

The SMB experiments were performed using a SEMBA Biosciences Octave 100 SMB

277 unit with four pumps, all of which were compatible with dichloromethane. The pump 
278 configuration is shown in Fig. 3. The 8 columns for the SMB experiments were obtained from

279 ACE Glass, Inc. and were $65 \mathrm{~cm}$ in length with an inner diameter of $2.54 \mathrm{~cm}$. HPLC analysis of

280 samples from the SMB experiments was accomplished using an Agilent 1100 HPLC with a 281 diode array detector and an auto-sampler. The column was an Inertsil ODS-2 HPLC column,

282 which was $150 \mathrm{~mm}$ in length, had an inner diameter of $4.6 \mathrm{~mm}$, and particle size of 5 microns.

283 Batch SEC chromatography experiments were accomplished using a diode array detector 284 (Agilent 1260 DAD VL), two Agilent PrepStar SD-1 pumps, a manual injection system, and an 285 Agilent 440-LC fraction collector. SMB column packing that required recirculating solvent used 286 an IsmaTec IP 65 pump.

\subsection{Column Packing}

The XAD $1180 \mathrm{~N}$ resin was packed with sodium chloride and sodium carbonate salts in

289 the pores to prevent bacterial growth. These salts must be removed to access all the pore space in

290 the particles so the resin was washed with reverse-osmosis (RO) water using a resin to water

291 ratio of 1:1.5 under stirring conditions for over 3 hours to remove the salts from the particles.

292 The liquid was decanted, and the resin was washed two more times in the same manner to ensure

293 that the salts were removed. After washing, the resin was dried overnight at room temperature in

294 a fume hood and then weighed. Isopropanol (IPA) was added to the resin particles in a 1:1

295 volume mixture and sonicated for 30 minutes to removed bubbles from the porous particles. The

296 low density of IPA ensured that all the resin particles were completely submerged and IPA can

297 be easily displaced by the acetone/dichloromethane mixture which was used in later experiments.

298 The mixture was allowed to settle overnight. 
The dead volume for each column was determined by weighing the column caps dry and

300 then reweighing the caps after pumping RO water through the caps until air bubbles were no

301 longer produced.

302 Once the dry weights of all the parts of the column were obtained, a slurry, which 303 consists of equal volumes of resin and IPA, was poured into the column with one end fitting 304 attached at the column outlet without a plug, so the IPA was allowed to flow out of the column.

305 When the top of the resin packing reached the top of the column, the bottom fitting was plugged 306 and the other end fitting of the column was attached and plugged. IPA was recycled using 307 downward flow at more than $30 \mathrm{~mL} / \mathrm{min}$. If the resin packing height was reduced, then more 308 resin/IPA slurry was added to the top of the column and the IPA recycle was repeated. If the 309 packing height did not change after more than 2 hours, the column was considered packed.

\subsection{Column Characterization}

To determine the interparticle bed void fraction $\left(\varepsilon_{b}\right), 10 \mathrm{~mL}$ pulses of $0.5 \mathrm{~g} / \mathrm{L}$ blue dextran

312 in 50/50 IPA/water were detected at a wavelength of $500 \mathrm{~nm}$. The flowrate was $5 \mathrm{~mL} / \mathrm{min}$ with a

313 downward flow direction. After the bed void fraction was determined, the solvent in the columns

314 was exchanged for 50/50 (vol.\%) DCM/ACE.

315 The total void fraction $\left(\varepsilon_{t}\right)$ was determined from long pulses of RDP since it was small

316 enough to completely penetrate all the pores of the particles. Long pulses were performed by

317 feeding 1-1.3 column volumes (CV) of the polymer or flame retardant solution to the column,

318 and then changing the feed to clean eluent to wash the column. The resulting breakthrough and

319 wash curves were used to determine size exclusion factors of the different components. VERSE

320 simulations of the experiments were used to determine apparent pore diffusivities for each

321 component by fitting the simulations to the experimental data. Initial estimates for the pore 
322 diffusivities were obtained from the Brownian diffusivities $\left(D_{\infty}\right)$ and the Mackie-Meares 323 correlation [51] (Supplementary Materials, S.3). The flowrate was $5 \mathrm{~mL} / \mathrm{min}$ in downward flow.

324 Since the mobile phase was 50\% ACE by volume, SAN, RDP, and BPADP could not be 325 monitored by the UV detector because of the large absorbance of ACE at all detectable 326 wavelengths. To obtain breakthrough curves, effluent samples were collected periodically and 327 analyzed by HPLC. on the principle of gradient polymer elution (GPEC) chromatography [28]. In GPEC, the sample

331 was injected into a poor solvent for the components so that the components precipitated on the

332 solid phase [52]. The mobile phase was then gradually changed to become a stronger solvent for 333 the components, such that the components would redissolve at different mobile phase 334 compositions. The different solubilities of the components in different mobile phase 335 compositions provided the necessary separation for the components to be detected by a UV 336 detector. used for detection was $260 \mathrm{~nm}$. The column was heated to $32^{\circ} \mathrm{C}$ and the injection volume was set 339 to $10 \mu \mathrm{L}$. The flowrates and solvent gradient are shown in Table S2.1 in the Supplementary 340 Material.

\subsection{SMB Fast Startup}

The SEMBA system allowed for a feed solution to be pumped through specific columns

343 and then sent to waste. For each preloading, a solution was pumped at $15 \mathrm{~mL} / \mathrm{min}$ through a 344 single column for 20 minutes. During this period, effluent samples were taken at 11, 13, 15, 17, 
345 and 19 minutes after the start of the loading. If the solution contained SAN, sampling times were 346 added at 7 and 9 minutes. The samples were analyzed using HPLC to obtain breakthrough

347 curves, which can be compared with VERSE simulations to verify the parameters.

348 After the 20 minutes had elapsed, the SEMBA unit added the next column in series to the

349 original column. The same solution was again pumped at $15 \mathrm{~mL} / \mathrm{min}$ for 20 minutes with the

350 same sampling schedule. After two columns had been loaded, the solution was changed and the 351 process was repeated for the next set of columns.

352 This procedure was performed for Runs 1, 4, and 6. For Runs 4 and 6, an elution step was 353 added after the three sets of columns were loaded. Clean eluent was pumped through all 8 354 columns connected in series at $5 \mathrm{~mL} / \mathrm{min}$ for 20 minutes. This elution step shifted the 355 concentration profiles into the same positions as the steady-state concentration profiles, which 356 reduced the amount of time for the SMB experiments to reach cyclic steady state.

\subsection{SMB Operation}

The feed solutions were made by dissolving SAN, RDP, or BPADP in 50/50 DCM/ACE

359 by volume. The eluent was clean 50/50 ACE/DCM by volume. The pump flowrates and

360 switching time were determined from the Speedy Standing Wave Design (SSWD) method and 361 set in the SEMBA program.

362 Glass bottles $(\sim 100 \mathrm{~mL})$ were used to collect the extract and raffinate product streams for 363 HPLC analysis. Immediately after a switch, a set of bottles (Set 1) was substituted with a new set 364 of bottles (Set 2) while the currently full set was weighed. Samples of roughly $20 \mathrm{~mL}$ were taken 365 from the bottles for archival purposes. Small $(\sim 1 \mathrm{~mL})$ samples were taken and analyzed by 366 HPLC to determine the polymer concentrations in each product stream. Extract samples were 367 diluted to $50 \%$ of the original concentration to keep RDP concentrations within the linear region 
368 of the established calibration curve. The bottles of Set 1 were then emptied, rinsed with DCM,

369 and dried before replacing the bottles of Set 2 after the next switch.

\section{$370 \quad 4.0$ Results}

\subsection{Intrinsic Parameters for SSWD and VERSE Simulations}

373 packing and characterization results are presented in Table 4, along with numerical parameters

374 used in VERSE simulations. Average particle size was provided by the manufacturer. Bed void $375\left(\varepsilon_{b}\right)$ and total void $\left(\varepsilon_{t}\right)$ fractions were determined using blue dextran pulses and RDP frontals, 376 respectively. Particle porosity $\left(\varepsilon_{p}\right)$ was determined from the other two void fractions. The dead 377 volume $(D V)$, in terms of percent of one column volume (CV), includes the system dead volume 378 per $\mathrm{CV}$ and the dead volume in the caps of a column.

379 Apparent pore diffusivities $\left(D_{p}\right)$ and size-exclusion factors $\left(K_{s e}\right)$ listed under the Batch 380 column were estimated from column frontal data. The $K_{s e}$ values of SAN and BPADP were fine381 tuned using SMB data and are listed under the SMB column.

\subsection{Column Characterization}

Chromatograms of the component frontals and blue dextran pulses are shown in Fig. 5.

384 The mass center of the blue dextran pulse gives the bed void fraction (0.37). The component 385 frontals were fit with VERSE simulations to determine their size-exclusion factors and 386 diffusivities, which are reported in Table 4. The estimated intraparticle diffusivities are less than $38710 \%$ of the Brownian diffusivities. These ratios are similar to those of other polymeric resins 388 reported in the literature [44]. 


\subsection{Experimental Testing of SSWD and Fast Startup Methods}

The intrinsic parameters obtained from batch chromatography in Table 4 were used to

391 design the SMB flowrates and switching times for Runs 1-3. After these runs, the parameters

392 were fine-tuned by comparing the VERSE column profiles with experimental profiles near cyclic

393 steady-state to fit the experimental data. The SMB design for Run 4 was obtained using the new

394 parameters. Fast startup methods based on the design of Run 4 were compared to the literature

395 fast startup method and to startup from clean columns. Run 5 was designed to separate a ternary

396 mixture. The results were used to fine-tune the size-exclusion factor for BPADP, which was

397 overestimated from batch SEC experiments. The fine-tuned size-exclusion factor was used to

398 design Run 6. Summaries of the SMB designs (operating parameters) and experimental results

399 are presented in Tables 5 and 6, respectively.

\subsection{SMBs for Separating SAN from RDP (Runs 1-4)}

\subsubsection{SMB Run 1}

402

The first SMB run was designed for $95 \%$ yield of each component and to verify the 403 estimated intrinsic parameters. To quickly reach cyclic-steady-state, the feed flowrate was set at $404 \quad 0.6 \mathrm{~mL} / \mathrm{min}$ so that the switching time would be relatively short ( 37 $\mathrm{min})$.

To quickly reach steady-state, the columns were preloaded with SAN/RDP solutions to 406 approximate the final steady state column profile predicted from the VERSE chromatography 407 simulation software. The feed solutions were 3 wt.\% RDP for columns 1 and 2, 3 wt.\% RDP 408 with 2 wt. $\%$ SAN for columns 3 and 4, and 2 wt.\% SAN for columns 5 and 6 . The preloaded 409 column profiles obtained from a rate-model based simulation program (VERSE) are shown in 410 Fig. 6a. 
412 Fig. 6. The simulated SAN profile lagged behind the experimentally obtained profile, Fig. 6 b.

413 The $K_{s e, S A N}$ was reduced from 0.63 to 0.61 to better fit the simulated SAN profile to the

414 experimental results, Fig. 6c. The raffinate history of Run 1 shows periodic fluctuations, which

415 resulted from the recycle flowrate being set to zero on the fifth step of every cycle. The error was

416 corrected after the $24^{\text {th }}$ step (886 minutes) and the raffinate concentration stabilized very quickly

417 to the value predicted by VERSE simulation.

418 The experimental RDP concentrations on the plateau are lower than the simulation 419 results, which is most likely due to some dilution of the profile samples because of the dead 420 volume in the sampling tubing. There also may have been some errors in diluting the profile 421 samples for HPLC measurement.

\subsubsection{SMB Run 2}

The second SMB experiment did not have a cleaning step between experiments, so the

424 column profile in Fig. 6b was used as the initial column profile for Run 2 in simulations. The

425 feed for Run 2 was also 5 wt.\% of each component, but the desired yield was increased to $97 \%$.

426 The effluent histories and column profiles at the end of 51 steps are shown in Fig. 7.

427 The simulated column profiles values lagged behind the experimental profiles when the 428 column length was assumed to be $65 \mathrm{~cm}$, Fig. 7a. When the packing length was changed to 63 $429 \mathrm{~cm}$, the simulation results fit the experimental data much better, Fig. 7b. The error in packing 430 length appears more prominently in Run 2 because it was run for a larger number of steps $(\sim 84$, 431 in total) compared to Run 1 (33 steps). The small error ( 3\%) in packing length could be from a 432 small error in estimating the bed void fraction or column dead volume, such that the effective 433 packing length was $2 \mathrm{~cm}$ shorter than the nominal length. It could also be due to small errors in 
434 the length or inner diameter of the glass columns. The rest of the simulations were based on a 435 packing length of $63 \mathrm{~cm}$. The RDP wave was more affected by the column length change than 436 the SAN wave because the size-exclusion factor of RDP is larger than that of SAN. Reducing the 437 column length from $65 \mathrm{~cm}$ to $63 \mathrm{~cm}$ (3\% difference) advances the edge of the RDP trailing wave 438 in Zone I by the difference in retention (i.e. $0.03 * L_{c} *$ retention factor $(0.69) * 51$ steps $\approx 1 L_{c}$ ). 439 The same reasoning predicts that the SAN waves should differ by $\sim 0.6 \mathrm{~L}_{\mathrm{c}}$ (retention factor $=$ 440 0.42). However, the differences for edges of the advancing waves in Zone IV are reduced 441 because the SWD focuses them toward the raffinate port.

442 The initial column profile of Run 2 was the final profile of Run 1 and the desorbent port 443 was located at the inlet of Column 1 (in SEMBA notation). As the operating conditions changed 444 to Run 2, the default location of the desorbent port in SEMBA was the inlet of Column 1. Instead 445 of entering Column 2 as intended, the desorbent again entered Column 1 at the beginning of Run

446 2. This resulted in low RDP concentration in the extract at the start of the experiment. As SMB 447 operation continued, the concentration waves recovered to their cyclic-steady-state positions. 448 The column profiles at the end of Run 2 agreed with the predicted values from simulation. The 449 effluent histories were well predicted by VERSE, which took into account the port location at the 450 beginning of Run 2.

\subsubsection{SMB Run 3}

452 Run 3 also did not have a cleaning step, so the initial column profile for Run 3 was the 453 final profile of Run 2 in the simulation. Run 3 was a repeat of the designed operating conditions 454 for Run 1, which were determined from SSWD based on the nominal column length of $65 \mathrm{~cm}$. 455 The effluent histories and column profiles at the end of step 56 are shown in Fig. 8. The 456 sharp rise in the RDP concentration around step 17 was most likely due to a flowrate problem 
caused by the desorbent pump, which produced a lower flowrate than desired. This problem

458 persisted for 3 steps before it could be corrected. After correction, RDP concentrations slowly 459 approached the steady-state value.

460 From the data obtained from Runs 1-3, the average column length was determined to be $46163 \mathrm{~cm}$ instead of $65 \mathrm{~cm}$ and the size exclusion factor for SAN was reduced to 0.61 from 0.63.

462 The designs of Runs 4 and 6 were based on these new parameters.

\subsubsection{SMB Run 4}

Run 4 was designed to separate RDP from SAN with higher feed concentrations (7 wt.\%

465 each) and a higher yield requirement (99\%) compared to the previous runs. The columns were

466 washed with pure solvent after Run 3. The first preloading strategy (VERSE) was used to

467 approximate the steady-state column profile. The preloading solutions were $6.5 \mathrm{wt} \%$ RDP for

468 columns 1 and 2 (Zone I), 6.5 wt \% RDP with 5.7 wt \% SAN for columns 3 and 4 (Zone II), and

4695.7 wt \% SAN for columns 5 and 6 (Zone III). All eight columns were then connected in series

470 and eluent was pumped at $5 \mathrm{~mL} / \mathrm{min}$ for 20 minutes. This elution step shifted the concentration

471 waves into their steady-state positions. The preloaded column profiles obtained from VERSE are

472 shown in Fig. 9a. Dips in concentrations on the plateaus are due to incomplete saturation of the

473 columns before elution.

474 The column profiles and effluent histories of Run 4 at the end of step 27 are shown in

475 Fig. 9b. The extract flowrate in the first two steps was lower than the set flowrate. After the 476 flowrate was corrected, the effluent data agreed with simulation results. The purities for both 477 streams were greater than 99\%. The experimental product concentrations, purities (>99\%), and 478 yields (>99\%) agreed closely with those of SWD. This preloading strategy effectively shortened 
479 the time required for the product concentrations to reach $95 \%$ of their cyclic steady-state values 480 to 3 steps.

\subsection{Comparison of Fast Startup Methods}

Once the first fast startup method was verified with experiments, it was compared to

483 other fast startup methods and startup from clean columns. Run 4 was used as the base case and

484 VERSE simulations were used to determine the startup time for each method. The results of the

485 VERSE simulations of SMB Run 4 are shown in Fig. 10. A clean startup of Run 4 was simulated

486 for 80 steps. The product concentrations after 80 steps were determined to be the cyclic steady-

487 state values. All the preloading strategies achieved cyclic steady state in fewer steps than the 488 regular startup from clean columns, which took over 36 steps ( $>4$ cycles).

489 The literature method of preloading four columns with the feed solution was the easiest to 490 implement, but has a longer startup time than the two proposed methods. The extract product had 491 very low purity until after 15 steps and the raffinate product did not reach $95 \%$ of the cyclic 492 steady-state value until after 29 steps. However, this strategy does not require already pure 493 solutions for preloading and can be beneficial when first starting up a process.

494 Within one step, the product concentrations of the first (VERSE) preloading strategy 495 reached $95 \%$ of their cyclic steady-state values and the product purities were greater than $99 \%$.

496 This preloading strategy allows for continuous product withdrawal from the first step, unlike the 497 literature method.

498 The second (SWD) strategy can be used when simulation software, such as VERSE, is 499 unavailable. This strategy uses the SWD to determine the four zone flowrates for a given feed 500 flowrate and specified yield. Once the zone flowrates are specified, the product flowrates can be 501 calculated and an overall mass balance can be performed to determine the product 
502 concentrations. These concentrations are used for the preloading solutions. However, the first

503 strategy is more general and can take into account components which are allowed to distribute

504 between the product ports. The two preloading strategies give similar effluent histories and 505 reduce the startup time by more than 31 fold.

\subsection{SMBs for Separating SAN from RDP/BPADP (Runs 5-6)}

\subsubsection{SMB Run 5}

A second flame retardant (BPADP) was added to the feed mixture for Run 5. Because the

509 two flame retardants (FRs) can be recovered together, the separation is pseudo-binary. The two

510 flame retardants were expected to migrate at the same speed in SMB, based on the batch

511 experiments. Thus the operating parameters for Run 5 were the same as Run 2. The effluent

512 histories are presented in Fig. 11.

513 Some BPADP was found in the raffinate and its concentration in the Extract was lower

514 than that of RDP, most likely due to the overestimation of the size-exclusion factor of BPADP.

515 Column profiles were not taken because raffinate flowrate was found to be lower than the set

516 value and leaks were also observed near the end of the experiment. The results indicated that

517 BPADP migrated faster than predicted and the $K_{s e, B P A D P}$ was overestimated from batch tests. By

518 reducing $K_{S e, B P A D P}$ from 1.0 to 0.96 , the simulated raffinate and extract histories of all 519 components agreed closely with the experimental histories over 50 steps, Fig. 11.

\subsubsection{SMB Run 6}

521 The revised parameters for BPADP were used in the SSWD to obtain the operating 522 conditions of Run 6 . The columns were washed with pure solvent, then preloaded using the first 523 preloading method to approximate the steady-state column profiles. 
525 RDP and 4 wt \% BPADP with 4 wt \% SAN for Zone II, and 4 wt \% SAN for Zone III. All eight

526 columns were then connected in series and eluent was pumped through at $5 \mathrm{~mL} / \mathrm{min}$ for 20

527 minutes. This elution step shifted the solute bands into their steady-state positions, Fig. 12a. The

528 transient column profiles from the preloaded columns to the start of step 40 are shown in Fig.

529 12a-f. The simulated effluent histories and column profiles at the end of step 40 are compared

530 with experimental data in Fig. 12g.

531 The extract and raffinate histories agree with simulations and reach $95 \%$ of their steady-

532 state values within 2 steps (37 steps if starting from clean columns). The preloading strategy

533 reduced the startup time by more than 18 fold. The purities of both streams are also very high

$534 \quad(\sim 100 \%$ for the extract, $98 \%$ for the raffinate) and agree closely with those from SSWD.

\subsection{SSWD Optimization of a Large-Scale SEC-SMB}

After the intrinsic parameters were fine-tuned and verified with pilot SMB experiments,

537 SSWD was used to optimize a large scale SEC-SMB for the lowest unit separation cost while

538 satisfying an imposed pressure limitation. The production was scaled to 10,000 tonnes of

539 FR/year and the maximum pressure drop was set at 100 psi per zone. The feed concentration was

540 fixed at $10 \mathrm{wt} \% \mathrm{FR}$ and $10 \mathrm{wt} \% \mathrm{SAN}$ and the viscosity was estimated to be $100 \mathrm{cP}$ [53]. The 15

541 decision variables that can be optimized are column configuration (4), column length (1), dead

542 volume (1), yields (3), particle size (1), and operating parameters (5). As discussed in Section

$5432.3, D V$ was fixed at $1.9 \%$ of the total column volume. The total number of columns was varied

544 from eight to twelve. In order for the true moving bed assumption to apply to SMBs, a constraint

545 of two or more columns per zone was placed on the column configuration [41]. Because of the

546 high feed viscosity, the column length allowed by the pressure limit is relatively short. In order 
547 to obtain a practical column length, the minimum column length was set to be $0.5 \mathrm{~m}$. Because the

548 FRs must be recovered with high purity, the yields were set to be $99 \%$ or higher for each

549 component. The particle size was allowed to vary from 0.5-2.0 times the experimental particle

550 radius.

The SSWD equations for the ternary separation of FRs from SAN were used to determine

552 solvent consumption and sorbent productivity for a given SEC-SMB design. These performance

553 criteria combined with cost functions (see Appendix B) can generate total separation costs for a

554 large number of SEC-SMB designs by systematically varying the decision variables. The values

555 of the decision variables which result in the lowest total separation cost were found by the

556 algorithm shown in Fig. C.1.

557 The optimal particle size was found to be $112 \mu \mathrm{m}$, which was the smallest particle size

558 examined. Assuming the resin cost is independent of particle size, smaller particles result in

559 higher productivity and lower cost. Because of the high feed viscosity, the column length is

560 already relatively short $(0.56 \mathrm{~m})$. Decreasing the particle size further would shorten the column

561 length to below $0.5 \mathrm{~m}$.

562 The column configuration did not have a large effect on the overall separation cost.

563 Adding more columns further reduces the column length. In order to obtain a column length

564 larger than $0.5 \mathrm{~m}$, the optimum column configuration was found to be 2-2-2-2.

565 The optimal yields were found to be $99 \%$ for each component. Increasing the yields of

566 the components increases the total cost of the separation, with a very sharp increase in cost when 567 yields approach $99.9 \%$.

568 When particle size, column configuration, and yields are fixed at the optimal values, the 569 total cost can be plotted against two key dimensionless groups, $P_{e b}{ }^{*}$ and $N_{D}{ }^{*}$. This surface gives 
570 an overview of how total cost varies with column length $\left(P_{e b}{ }^{*}\right)$ and step time $\left(N_{D}{ }^{*}\right.$, Eq. (2)) and

571 how a pressure limit constrains column length and step time. Because the total cost surface is

572 concave up, the inverse of total cost is plotted for convenience in Fig. 13a. The highest point in

573 Fig. 13a corresponds to the design with the lowest separation cost.

$574 \quad$ The dark grey surface represents the system's pressure limit. This surface was produced

575 using Eq. (6). Particle size, pressure limit, phase ratio, and viscosity are fixed. The column length

576 in the denominator of the left hand side of Eq. (6) can be replaced with the Peclet number, Eq.

577 (A.13b), and the port velocity can be replaced by $N_{D}{ }^{*}$, Eq. (2). Since the material properties are

578 fixed, Eq. (6) is only a function of $P_{e b}{ }^{*}$ and $N_{D}{ }^{*}$. Equation (6) can be rearranged to have the left

579 hand side be a constant and the right hand side be a function of $P_{e b}{ }^{*}$ and $N_{D}{ }^{*}$, which results in a

580 pressure limit line, Fig. 13b. To visualize the designs which will satisfy the pressure limit, this

581 pressure limit line is extended in the cost dimension as a surface, Fig. 13a. The intersection of the

582 pressure surface and the cost surface represents the combinations of $L_{c}\left(P_{e b}{ }^{*}\right)$ and zone velocities

$583\left(N_{D}{ }^{*}\right)$ that give the pressure drop in Zone I equal to $\Delta P_{\max }$. Designs to the left of the surface,

584 smaller $P_{e b}{ }^{*}$ (shorter $L_{c}$ ) or larger $N_{D}{ }^{*}$ (slower zone velocities), will satisfy the pressure

585 requirement. Lines of constant total cost are plotted in Fig. 13b along with the pressure limit

586 curve. The minimum cost design which satisfies the pressure limitation is marked with an "x."

587 Figure 14 shows the equipment, solvent, sorbent, and total costs plotted against $N_{D}^{*}$ at the

588 optimum $P_{e b}^{*}(\sim 353)$. The vertical black line represents the pressure limit and designs to the left

589 of the line do not satisfy the pressure requirement. Because the material properties are fixed, the

590 column length can be calculated from $P_{e b}^{*}$ and the port velocity can be calculated from $N_{D}^{*}$.

591 Estimated costs for separating FRs from SAN at a scale of 10,000 tonnes of FRs/year for

592 the optimized SEC-SMB and batch SEC elution are shown in Table 7. Given the same feed and 
593 product requirements, the unit separation cost of SEC-SMB is only $2.6 \%$ of that of conventional

594 batch elution SEC. Since the separation cost is less than $10 \%$ of the purchase cost of these FRs, 595 this technology is economically attractive at this scale.

\section{$596 \quad 5.0$ Conclusions}

The SSWD theory has been extended to ternary mixtures and was verified by SEC-SMB experiments. SEC-SMB can produce high purity FRs and SAN with high yield from one of the

599 side streams of the SEPoR process. The results of this study show that intrinsic parameters

600 estimated from single column experiments can have a small (a few \%) errors. The operating flow

601 rates and step time based on the estimated parameters can result in significant deviations of

602 column profiles or product purities in long SMB operations. Pilot SMB experiments with 10 or

603 more cycles are needed to detect any small errors. Comparison of VERSE simulated column

604 profiles and effluent histories with the pilot SMB data can help obtain accurate parameters,

605 which are needed for designing reliable SMBs for large-scale production. Fast startup methods

606 based on SWD and VERSE effectively reduced the startup time for the SMB by more than 18

607 fold. The estimated separation cost for FR recovery by SEC-SMB is less than 3\% of that for

608 batch SEC and is less than $10 \%$ of the FR purchase price. The results of this study may help

609 develop other SEC-SMB processes for recycling applications, which require high-purity 610 products.

\subsection{Acknowledgments}

612 We would like to thank Mingzhe Chong, Shinae Kim, Matthew Nieves, Ye Tao, and Dr.

613 Wei Dai assisted with HPLC analyses and batch column experiments. Dr. David Zoller at 
614 SABIC Innovative Plastics assisted with analysis of polymer samples and helped set up the 615 HPLC analysis for the samples obtained from the SMB experiments at SABIC. Dr. Zheng Liu at 616 SABIC-IP helped with sample collection during SMB experiments at SABIC. Dr. Philip Bell 617 offered industrial guidance and helped with sample collection during SMB experiments. This 618 work was funded in part by the Hugh and Edna Donnan Dissertation Fellowship (Purdue 619 University), the School of Chemical Engineering (Purdue University), the National Science 620 Foundation (CBET 1403854), and SABIC Innovative Plastics, Mt. Vernon, IN. 


\section{References}

622 [1] T. Wakolbinger, F. Toyasaki, T. Nowak, A. Nagurney, When and for whom would ewaste be a treasure trove? Insights from a network equilibrium model of e-waste flows, Int. J. Prod. Econ. 154 (2014) 263-273. doi:10.1016/j.ijpe.2014.04.025.

[2] X. Yang, L. Sun, J. Xiang, S. Hu, S. Su, Pyrolysis and dehalogenation of plastics from waste electrical and electronic equipment (WEEE): a review., Waste Manag. 33 (2013)

[3] R. Rajarao, R. Ferreira, S.H.F. Sadi, R. Khanna, V. Sahajwalla, Synthesis of silicon

[4] B.H. Robinson, E-waste: an assessment of global production and environmental impacts., Sci. Total Environ. 408 (2009) 183-91. doi:10.1016/j.scitotenv.2009.09.044.

S. Herat, P. Agamuthu, E-waste: a problem or an opportunity? Review of issues,

[6] S. Schwarzer, A.D. Bono, G. Giuliani, S. Kluser, P. Peduzzi, E-waste, the hidden side of

[7] J. Beigbeder, D. Perrin, J.-F. Mascaro, J.-M. Lopez-Cuesta, Study of the physico-chemical properties of recycled polymers from waste electrical and electronic equipment (WEEE) sorted by high resolution near infrared devices, Resour. Conserv. Recycl. 78 (2013) 105114. doi:10.1016/j.resconrec.2013.07.006. management systems in the United States, Resour. Conserv. Recycl. 52 (2008) 955-964. doi:10.1016/j.resconrec.2008.03.002. Hafner, et al., Plastic accumulation in the North Atlantic subtropical gyre., Science. 329 (2010) 1185-8. doi:10.1126/science.1192321. 
656

657

658

659

660

661

662

663

664

665

666

667

668

669

670

671

672

673

674

675

676

677

678

679

680

681

682

683

684

685

686

687

688

689

690

691

[12] C.M. Rochman, M.A. Browne, B.S. Halpern, B.T. Hentschel, E. Hoh, H.K. Karapanagioti, et al., Policy: Classify plastic waste as hazardous., Nature. 494 (2013) 169-71.

doi:10.1038/494169a.

[13] D. Lithner, A. Larsson, G. Dave, Environmental and health hazard ranking and assessment of plastic polymers based on chemical composition., Sci. Total Environ. 409 (2011) 330924. doi:10.1016/j.scitotenv.2011.04.038.

[14] G.S. Weeden Jr, N.H. Soepriatna, P. Bell, N.-H.L. Wang, Methods useful for recovering polymers from electronic and other wastes, PCT/US2014/37824, 2014.

[15] G.S. Weeden, N.H. Soepriatna, N.-H.L. Wang, Method for Efficient Recovery of HighPurity Polycarbonates from Electronic Waste, Environ. Sci. Technol. 49 (2015) 24252433. doi:10.1021/es5055786.

[16] D. Bright, S. Dashevsky, Resorcinol Bis (Diphenyl Phosphate), a Non-Halogen FlameRetardant Additive, J. Vinyl Addit. Technol. 3 (1997) 170-174. http://onlinelibrary.wiley.com/doi/10.1002/vnl.10184/abstract (accessed May 28, 2014).

[17] A. Ballesteros-Gómez, S.H. Brandsma, J. de Boer, P.E.G. Leonards, Analysis of two alternative organophosphorus flame retardants in electronic and plastic consumer products: Resorcinol bis-(diphenylphosphate) (PBDPP) and bisphenol A bis (diphenylphosphate) (BPA-BDPP), Chemosphere. 116 (2014) 10-14. doi:10.1016/j.chemosphere.2013.12.099.

[18] I.C. Nnorom, O. Osibanjo, Sound management of brominated flame retarded (BFR) plastics from electronic wastes: State of the art and options in Nigeria, Resour. Conserv. Recycl. 52 (2008) 1362-1372. doi:10.1016/j.resconrec.2008.08.001.

[19] I. van der Veen, J. de Boer, Phosphorus flame retardants: Properties, production, environmental occurrence, toxicity and analysis, Chemosphere. 88 (2012) 1119-1153. doi:10.1016/j.chemosphere.2012.03.067.

[20] M. García-López, I. Rodríguez, R. Cela, Evaluation of liquid-liquid microextraction using polypropylene microporous membranes for the determination of organophosphorus flame retardants and plasticizers in water samples, Anal. Chim. Acta. 625 (2008) 145-153. doi:10.1016/j.aca.2008.07.026.

[21] Z. Gao, Y. Deng, W. Yuan, H. He, S. Yang, C. Sun, Determination of organophosphorus flame retardants in fish by pressurized liquid extraction using aqueous solutions and solidphase microextraction coupled with gas chromatography-flame photometric detector, J. Chromatogr. A. 1366 (2014) 31-37. doi:10.1016/j.chroma.2014.09.028.

[22] G.A. MacKay, R.M. Smith, Supercritical fluid extraction and chromatography?mass spectrometry of flame retardants from polyurethane foams, Analyst. 118 (1993) 741. doi:10.1039/an9931800741. 
692

693

694

695

696

697

698

699

700

701

702

703

704

705

706

707

708

709

710

711

712

713

714

715

716

717

718

719

720

721

722

723

724

[23] S.H. Smith, L.T. Taylor, Extraction of various additives from polystyrene and their subsequent analysis, Chromatographia. 56 (2002) 165-169. doi:10.1007/BF02493206.

[24] Y. Ma, K. Cui, F. Zeng, J. Wen, H. Liu, F. Zhu, et al., Microwave-assisted extraction combined with gel permeation chromatography and silica gel cleanup followed by gas chromatography-mass spectrometry for the determination of organophosphorus flame retardants and plasticizers in biological samples, Anal. Chim. Acta. 786 (2013) 47-53. doi:10.1016/j.aca.2013.04.062.

[25] B. Kowalski, M. Mazur, The Simultaneous Determination of Six Flame Retardants in Water Samples Using SPE Pre-concentration and UHPLC-UV Method, Water, Air, Soil Pollut. 225 (2014). doi:10.1007/s11270-014-1866-4.

[26] M. García-López, I. Rodríguez, R. Cela, Pressurized liquid extraction of organophosphate triesters from sediment samples using aqueous solutions, J. Chromatogr. A. 1216 (2009) 6986-6993. doi:10.1016/j.chroma.2009.08.068.

[27] E.M. Reingruber, A. Chojnacka, E. Jellema, B. de Bruin, W. Buchberger, P.J. Schoenmakers, Chromatographic examination of the chemical composition and sequence distribution of copolymers from ethyl and benzyl diazoacetate., J. Chromatogr. A. 1255 (2012) 259-66. doi:10.1016/j.chroma.2012.02.059.

[28] E. Uliyanchenko, S. van der Wal, P.J. Schoenmakers, Challenges in polymer analysis by liquid chromatography, Polym. Chem. 3 (2012) 2313. doi:10.1039/c2py20274c.

[29] D. Broughton, Molex: Case history of a process, Chem. Eng. Prog. 64 (1968) 60-65.

[30] D. Broughton, C.G. Gerhold, Continuous sorption process employing fixed bed of sorbent and moving inlets and outlets, 2,985,589, 1961.

[31] T. Kröber, M.W. Wolff, B. Hundt, a. Seidel-Morgenstern, U. Reichl, Continuous purification of influenza virus using simulated moving bed chromatography, $\mathrm{J}$. Chromatogr. A. 1307 (2013) 99-110. doi:10.1016/j.chroma.2013.07.081.

[32] P. Nestola, R.J.S. Silva, C. Peixoto, P.M. Alves, M.J.T. Carrondo, J.P.B. Mota, Adenovirus purification by two-column, size-exclusion, simulated countercurrent chromatography, J. Chromatogr. A. 1347 (2014) 111-121.

doi:10.1016/j.chroma.2014.04.079.

[33] M. Wellhoefer, W. Sprinzl, R. Hahn, A. Jungbauer, Continuous processing of recombinant proteins: Integration of refolding and purification using simulated moving bed size-exclusion chromatography with buffer recycling, J. Chromatogr. A. 1337 (2014) 48-56. doi:10.1016/j.chroma.2014.02.016. 
[34] Y. Xie, S. Mun, J. Kim, N.-H.L. Wang, Standing wave design and experimental validation of a tandem simulated moving bed process for insulin purification., Biotechnol. Prog. 18 (2002) 1332-44. doi:10.1021/bp025547r.

[35] S. Mun, Y. Xie, N.-H.L. Wang, Residence time distribution in a size-exclusion SMB for insulin purification, AIChE J. 49 (2003) 2039-2058. doi:10.1002/aic.690490814.

[36] Y. Xie, S.-Y. Mun, N.-H.L. Wang, Startup and Shutdown Strategies of Simulated Moving Bed for Insulin Purification, Ind. Eng. Chem. Res. 42 (2003) 1414-1425. doi:10.1021/ie020674d.

[37] S. Mun, Y. Xie, J.-H. Kim, N.-H.L. Wang, Optimal Design of a Size-Exclusion Tandem Simulated Moving Bed for Insulin Purification, Ind. Eng. Chem. Res. 42 (2003) 19771993. doi:10.1021/ie020680+.

[38] A. Geisser, T. Hendrich, G. Boehm, B. Stahl, Separation of lactose from human milk oligosaccharides with simulated moving bed chromatography., J. Chromatogr. A. 1092 (2005) 17-23. doi:10.1016/j.chroma.2005.03.061.

[39] M.-T. Liang, R.-C. Liang, Fractionation of polyethylene glycol particles by simulated moving bed with size-exclusion chromatography., J. Chromatogr. A. 1229 (2012) 107-12. doi:10.1016/j.chroma.2011.12.094.

[40] G. Biressi, O. Ludemann-Hombourger, M. Mazzotti, R.M. Nicoud, M. Morbidelli, Design and optimisation of a simulated moving bed unit: role of deviations from equilibrium theory., J. Chromatogr. A. 876 (2000) 3-15. http://www.ncbi.nlm.nih.gov/pubmed/10823498.

[41] Z. Ma, N.-H.L. Wang, Standing wave analysis of SMB chromatography: Linear systems, AIChE J. 43 (1997) 2488-2508. doi:10.1002/aic.690431012.

[42] B.J. Hritzko, Y. Xie, R.J. Wooley, N.-H.L. Wang, Standing-wave design of tandem SMB for linear multicomponent systems, AIChE J. 48 (2002) 2769-2787. doi:10.1002/aic.690481207.

[43] T. Mallmann, B. Burris, Z. Ma, N. Wang, Standing wave design of nonlinear SMB systems for fructose purification, AIChE J. 44 (1998). http://onlinelibrary.wiley.com/doi/10.1002/aic.690441206/abstract (accessed July 28, 2013).

[44] Y. Xie, C.A. Farrenburg, C.Y. Chin, S. Mun, N.-H.L. Wang, Design of SMB for a nonlinear amino acid system with mass-transfer effects, AIChE J. 49 (2003) 2850-2863. doi:10.1002/aic.690491117. 
772

773

774

775

776

777

778

779

780

781

782

783

784
[45] K. Lee, S. Mun, F. Cauley, G. Cox, N.-H.L. Wang, Optimal standing-wave design of nonlinear simulated moving bed systems for enantioseparation, Ind. Eng. Chem. Res. (2006) 739-752. http://pubs.acs.org/doi/abs/10.1021/ie0504248 (accessed July 28, 2013).

[46] K. Lee, C. Chin, Y. Xie, G. Cox, N.-H.L. Wang, Standing-wave design of a simulated moving bed under a pressure limit for enantioseparation of phenylpropanolamine, Ind. Eng. Chem. Res. (2005) 3249-3267. http://pubs.acs.org/doi/abs/10.1021/ie049413p (accessed July 28, 2013).

[47] G.S. Weeden, N.-H.L. Wang, Speedy standing wave design of size-exclusion simulated moving bed: Solvent consumption and sorbent productivity related to material properties and design parameters, J. Chromatogr. A, Manuscr. under Rev. (2015).

[48] S. Ergun, Fluid flow through packed columns, Chem. Eng. Prog. 48 (1952) 89-94.

[49] J. Bentley, S. Li, Y. Kawajiri, Experimental validation of optimized model-based startup acceleration strategies for simulated moving bed chromatography, Ind. Eng. Chem. Res. 53 (2014) 12063-12076. doi:10.1021/ie500714p.

[50] S. Li, Y. Kawajiri, J. Raisch, A. Seidel-Morgenstern, Optimization of startup and shutdown operation of simulated moving bed chromatographic processes, J. Chromatogr. A. 1218 (2011) 3876-3889. doi:10.1016/j.chroma.2011.04.051.

[51] J. Mackie, P. Meares, The diffusion of electrolytes in a cation-exchange resin membrane. I. Theoretical, Proc. R. Soc. London. 232 (1955) 498-509. http://rspa.royalsocietypublishing.org/content/232/1191/498.short (accessed December 15, 2014).

[52] C.N. Pomeranz, S. V Olesik, Separation of poly-3-hydroxyvalerate-co-3-hydroxybutyrate through gradient polymer elution chromatography., J. Chromatogr. A. 1218 (2011) 79437. doi:10.1016/j.chroma.2011.08.065.

[53] R.S. Spencer, J.L. Williams, Concentrated solution viscosity of polystyrene, J. Colloid Sci. 2 (1947) 117-129. doi:10.1016/0095-8522(47)90015-9. 


\section{Appendices}

\section{Appendix A - SSWD Derivation}

The mass transfer correction term in Eq. (1) is given by Eq. (A.1),

789

$$
\Delta_{i}^{j}=\frac{\beta_{i}^{j}}{L^{j}\left(1+\phi \delta_{i}\right)}\left[E_{b, i}^{j}+\frac{\phi\left(\delta_{i} v\right)^{2}}{K_{i}^{j}}\right]
$$

790 where $L^{j}$ is the length of zone $\mathrm{j}$, which is equal to $L_{c}$ times the number of columns in zone $\mathrm{j}\left(N^{j}\right)$;

791 and $K_{i}^{j}$ is the overall mass transfer coefficient of component $\mathrm{i}$ in zone j. Equation (A.1) can be

792 substituted into Eq. (1) and the result can be solved for the zone velocities, Eq. (A.2).

$793 \quad u_{0}^{I}=\left(1+\phi \delta_{2}\right) v+\frac{\beta_{2}^{I}}{L^{I}}\left[E_{b, 2}^{I}+\frac{\phi\left(\delta_{2} v\right)^{2}}{K_{2}^{I}}\right]$

$794 \quad u_{0}^{I I}=\left(1+\phi \delta_{1}\right) v+\frac{\beta_{1}^{I I}}{L^{I I}}\left[E_{b, 1}^{I I}+\frac{\phi\left(\delta_{1} v\right)^{2}}{K_{1}^{I I}}\right]$

$795 \quad u_{0}^{I I I}=\left(1+\phi \delta_{2}\right) v-\frac{\beta_{2}^{I I I}}{L^{I I I}}\left[E_{b, 2}^{I I I}+\frac{\phi\left(\delta_{2} v\right)^{2}}{K_{2}^{I I I}}\right]$

$u_{0}^{I V}=\left(1+\phi \delta_{1}\right) v-\frac{\beta_{1}^{I V}}{L^{I V}}\left[E_{b, 1}^{I V}+\frac{\phi\left(\delta_{1} v\right)^{2}}{K_{1}^{I V}}\right]$

$797 \quad u_{F}=u_{0}^{I I I}-u_{0}^{I I}$

798 The feed velocity $\left(u_{F}\right)$ is determined by a mass balance at the feed port. The equations for

799 the $\beta$ terms are given by Eq. (A.3), which were derived from equations presented in Hritzko et al.

$800 \quad[42]$.

$801 \quad \beta_{2}^{I}=\ln \left[\frac{Y_{2}}{\left(1-Y_{2}\right)} \frac{\left(\frac{u_{0}^{I I I}}{\left.u_{0}^{I V}-1\right)}\right)}{\left(1-\frac{u_{0}^{I I}}{u_{0}^{I}}\right)}\right]$ 
$802 \beta_{1}^{I I}=\ln \left\{\frac{\left(\frac{u_{0}^{I}}{u_{0}^{I I}}-1\right)}{\left(1-Y_{1}\right)}\left[\frac{Y_{1}}{\left(1-\frac{u_{0}^{I V}}{u_{0}^{I I I}}\right)}-1\right]\right\}$

$803 \quad \beta_{2}^{I I I}=\ln \left\{\frac{\left(1-\frac{u_{0}^{I V}}{u_{0}^{I I I}}\right)}{\left(1-Y_{2}\right)}\left[\frac{Y_{2}}{\left(\frac{u_{0}^{I}}{u_{0}^{I I}}-1\right)}+1\right]\right\}$

$804 \quad \beta_{1}^{I V}=\ln \left[\frac{Y_{1}}{\left(1-Y_{1}\right)} \frac{\left(1-\frac{u_{0}^{I I}}{u_{0}^{I}}\right)}{\left(\frac{u_{0}^{I I}}{u_{0}^{I V}}-1\right)}\right]$

806 to evaluate the axial dispersion coefficients.

$807 \quad E_{b}^{j}=10 \varepsilon_{b} R_{p} u_{0}^{j}$

808 The ratios of the axial dispersion coefficients $\left(\Gamma^{j}\right)$ were determined by substituting Eq. (A.4) into

809 the definition of $\Gamma^{j}$ in Table 2 and the resulting equations were rearranged to solve for $\Gamma^{j}$, Eq.

810 (A.5).

$811 \quad \Gamma^{I}=\frac{1+\phi \delta_{2}}{1+\phi \delta_{1}-\frac{\phi\left(\delta_{2}-\delta_{1}\right)}{P_{e b}^{*}}\left(\frac{\beta_{1}^{I V}}{N^{I V}}+\frac{\beta_{2}^{I}}{N^{I}}\right)}$

$812 \quad \Gamma^{I I}=\frac{1+\phi \delta_{1}}{1+\phi \delta_{1}-\frac{\phi\left(\delta_{2}-\delta_{1}\right)}{P_{e b}^{*}}\left(\frac{\beta_{1}^{I V}}{N^{I V}}+\frac{\beta_{1}^{I I}}{N^{I I}}\right)}$

$813 \quad \Gamma^{I I I}=\frac{1+\phi \delta_{2}}{1+\phi \delta_{1}-\frac{\phi\left(\delta_{2}-\delta_{1}\right)}{P_{e b}^{*}}\left(\frac{\beta_{1}^{I V}}{N^{I V}}-\frac{\beta_{2}^{I I I}}{N^{I I I}}\right)}$

814 The overall mass transfer resistance, $\frac{1}{K_{i}^{j}}$, can be written explicitly as mass transfer

815 resistances in series for linear systems, as shown in Eq. (A.6), 
$816 \frac{1}{K_{i}^{j}}=\frac{R_{p}^{2}}{15 K_{s e, i} \varepsilon_{p} D_{p, i}}+\frac{R_{p}}{3 k_{f, i}}$

817 where $k_{f, i}$ is the film mass transfer coefficient. The last term on the right hand side of Eq. (A.6) is

818 negligible compared to intraparticle diffusion, or axial dispersion, for most low pressure systems.

819 The simplified Eq. (A.6) can be substituted into Eq. (A.2). The resulting equations can then be

820 simplified using the dimensionless groups in Table 2, Eq. (A.7).

$821 \quad u_{0}^{I}=v\left(1+\phi \delta_{2}+\frac{\beta_{2}^{I} \Gamma^{I}}{P_{e b}^{I V} N^{I}}+\frac{\phi^{2} \beta_{2}^{I} \delta_{2}{ }^{2}}{15 N_{D, 1} \lambda_{2} \gamma_{2} N^{I}}\right)$

822

$u_{0}^{I I}=v\left(1+\phi \delta_{1}+\frac{\beta_{1}^{I I} \Gamma^{I I}}{P_{e b}^{I V} N^{I I}}+\frac{\phi^{2} \beta_{1}^{I I} \delta_{1}^{2}}{15 N_{D, 1} N^{I I}}\right)$

$u_{0}^{I I I}=v\left(1+\phi \delta_{2}-\frac{\beta_{2}^{I I I} \Gamma^{I I I}}{P_{e b}^{I V} N^{I I I}}-\frac{\phi^{2} \beta_{2}^{I I I} \delta_{2}{ }^{2}}{15 N_{D, 1} \lambda_{2} \gamma_{2} N^{I I I}}\right)$

$u_{0}^{I V}=v\left(1+\phi \delta_{1}-\frac{\beta_{1}^{I V}}{P_{e b}^{I V} N^{I V}}-\frac{\phi^{2} \beta_{1}^{I V} \delta_{1}{ }^{2}}{15 N_{D, 1} N^{I V}}\right)$

825 An even more detailed derivation of the SSWD equations for separations of binary mixtures can

826 be found in Weeden and Wang [47].

827 Equations (A.3), (A.5), and (A.7) can be extended to separations of ternary mixtures. The 828 only differences are the component subscripts, which denote which component is confined in 829 that zone. If the fast solute (solute 1) is split from the other two (Fig. 3b), then the slowest solute

830 (solute 3 ) is controlled in Zone I, solute 1 is controlled in Zone II, solute 2 is controlled in Zone

831 III (and solute 3 is pinched), and solute 1 is controlled in Zone IV. The resulting zone velocity

832 equations are shown in Table 3, Eq. (4).

833 If solute 3 is split from the other two (Fig. 3c), then solute 3 is controlled in Zone I, solute

8342 is controlled in Zone II (and solute 1 is pinched), solute 3 is controlled in Zone III, and solute 1

835 is controlled in Zone IV. The resulting zone velocity equations are shown in Table 3, Eq. (5). 
837 balances. The desorbent and feed flowrates are given by Eq. (A.8) and Eq. (A.9), respectively,

$838 D=\varepsilon_{b} S\left(u_{0}^{I}-u_{0}^{I V}\right)$

$839 \quad F=\varepsilon_{b} S\left(u_{0}^{I I I}-u_{0}^{I I}\right)$

840 where $S$ is the column cross-sectional area.

841 One important factor in calculating the overall cost for SEC-SMB systems is the amount

842 of solvent used by the process. The solvent cost is related to the ratio of the desorbent flowrate

843 over the feed flowrate $(D / F)$, which is also an indicator of the dilution of the products. The

844 equation for $D / F$ for splitting solute 1 from solutes 2 and 3 is shown in Eq. (A.10a). The equation

845 for $D / F$ when splitting solute 3 from solutes 1 and 2 is shown in Eq. (A10b).

$846 \frac{D}{F}=\frac{u_{0}^{I}-u_{0}^{I V}}{u_{0}^{I I}-u_{0}^{I I}}=\frac{\frac{\left(\alpha_{3}-1\right)}{\left(\alpha_{2}-1\right)}+\frac{1}{P_{e b}^{*}}\left(\frac{\beta_{3}^{I} \Gamma^{I}}{N^{I}}+\frac{\beta_{1}^{I V}}{N^{I V}}\right)+\frac{1}{15 N_{D}^{*}}\left(\frac{\beta_{3}^{I} \alpha_{3}^{2}}{\lambda_{3} \gamma_{3} N^{I}}+\frac{\beta_{1}^{I V}}{N^{I V}}\right)}{1-\frac{1}{P_{e b}^{*}}\left(\frac{\beta_{2}^{I I} \Gamma^{I I I}}{N^{I I I}}+\frac{\beta_{1}^{I I} \Gamma^{I I}}{N^{I I}}\right)-\frac{1}{15 N_{D}^{*}}\left(\frac{\beta_{2}^{I I} \alpha_{2}{ }^{2}}{\lambda_{2} \gamma_{2} N^{I I I}}+\frac{\beta_{1}^{I I}}{N^{I}}\right)}$

$\frac{D}{F}=\frac{\frac{\left(\alpha_{3}-1\right)}{\left(\alpha_{3}-\alpha_{2}\right)}+\frac{1}{P_{e b}^{*}}\left(\frac{\beta_{3}^{I} \Gamma^{I}}{N^{I}}+\frac{\beta_{1}^{I V}}{N^{I V}}\right)+\frac{1}{15 N_{D}^{*}}\left(\frac{\beta_{4}^{I} \alpha_{3}^{2}}{\lambda_{3} \gamma_{3} \alpha_{2}^{2} N^{I}}+\frac{\beta_{1}^{I V}}{\alpha_{2}{ }^{2} N^{I V}}\right)}{1-\frac{1}{P_{e b}^{*}}\left(\frac{\beta_{3}^{I I} \Gamma^{I I I}}{N^{I I I}}+\frac{\beta_{2}^{I I} \Gamma^{I I}}{N^{I I}}\right)-\frac{1}{15 N_{D}^{*}}\left(\frac{\beta_{3}^{I I} \alpha_{3}^{2}}{\lambda_{3} \gamma_{3} \alpha_{2}^{2} N^{I I I}}+\frac{\beta_{2}^{I I}}{\lambda_{2} \gamma_{2} N^{I I}}\right)}$

Another important factor for calculating costs is the cost of the stationary phase (resin

849 cost). This cost is mainly affected by the system productivity $\left(P_{R, i}\right)$, which can be calculated

850 using Eq. (A.11),

$851 \quad P_{R, i}=\frac{S\left(u_{0}^{I I I}-u_{0}^{I I}\right) C_{F, i} Y_{i}}{S L_{c} N \phi \rho_{p}}$

852 where $\rho_{p}$ is the particle density (mass of stationary phase per particle volume). 
$855 P_{R, i}=\frac{Y_{i} C_{F, i}\left(\delta_{2}-\delta_{1}\right)}{N t_{s} \rho_{p}}\left[1-\frac{1}{P_{e b}^{*}}\left(\frac{\beta_{2}^{I I I} \Gamma^{I I I}}{N^{I I I}}+\frac{\beta_{1}^{I I} \Gamma^{I I}}{N^{I I}}\right)-\frac{1}{15 N_{D}^{*}}\left(\frac{\beta_{2}^{I I I} \alpha_{2}^{2}}{\lambda_{2} \gamma_{2} N^{I I I}}+\frac{\beta_{1}^{I I}}{N^{I I}}\right)\right]$

$856 P_{R, i}=\frac{Y_{i} C_{F, i}\left(\delta_{3}-\delta_{2}\right)}{N t_{s} \rho_{p}}\left[1-\frac{1}{P_{e b}^{*}}\left(\frac{\beta_{3}^{I I I} \Gamma^{I I I}}{N^{I I I}}+\frac{\beta_{2}^{I I} \Gamma^{I I}}{N^{I I}}\right)-\frac{1}{15 N_{D}^{*}}\left(\frac{\beta_{3}^{I I I} \alpha_{3}{ }^{2}}{\lambda_{3} \gamma_{3} \alpha_{2}{ }^{2} N^{I I I}}+\frac{\beta_{2}^{I I}}{\lambda_{2} \gamma_{2} N^{I I}}\right)\right]$

Since the Chung and Wen correlation is used to evaluate the axial dispersion coefficients,

858 the Peclet numbers can be simplified by substituting Eq. (A.4) into Eq. (3) and rearranged to

859 solve for $P_{e b}^{*}$, resulting in Eq. (A.13a).

$860 \quad P_{e b}^{*}=\frac{\phi\left(\delta_{2}-\delta_{1}\right)}{\left(1+\phi \delta_{1}\right)}\left[\frac{L_{c}}{10 \varepsilon_{b} R_{p}}+\frac{\beta_{1}^{I V}}{N^{I V}}\right]$

861 Equation (A.13a) can be rearranged to solve for $L_{c}$, Eq. (A.13b).

$862 L_{c}=10 \varepsilon_{b} R_{p}\left[\frac{P_{e b}^{*}\left(1+\phi \delta_{1}\right)}{\phi\left(\delta_{2}-\delta_{1}\right)}-\frac{\beta_{1}^{I V}}{N^{I V}}\right]$

Appendix B - Cost Functions

The total cost function used in this work to estimate the separation cost for SEC-SMB is

865 shown in Eq. (B.1).

866 Total Cost $(T C)=\operatorname{Resin} \operatorname{Cost}(R C)+$ Solvent Cost $(S C)+$ Equipment Cost $(E C)$

867 where all the costs have the units of $\$ / \mathrm{kg}$ FR.

868 The resin cost was calculated according to Eq. (B.2),

869

$R C\left[\frac{\$}{k g_{F R}}\right]=\frac{(\text { Vol.of resin })(\text { Resin price })}{(\text { Resin Life })(\text { Production })}=\frac{\left(\frac{N L_{C} \pi(I D)^{2}}{4}[L C V]\right)\left(19\left[\frac{\$}{L C V}\right]\right)}{(10[\text { years }])\left(10,000,000\left[\frac{k g_{F R}}{\text { year }}\right]\right)}$

870 where $N$ is the total number of columns and $I D$ is the column inner diameter.

871 The solvent cost was calculated according to Eq. (B.3),

872

$S C\left[\frac{\$}{k g_{F R}}\right]=\frac{\left(\frac{D}{F}\right)[\text { RR*recycle cost }+(1-R R) * \text { Solvent price }]}{(\text { Feed Conc. })(\text { Yield })}=\frac{\left(\frac{D}{F}\right)(.99 * .002+.01 * 1)[\$ / L]\left(1,000\left[\frac{g_{F R}}{k g_{F R}}\right]\right)}{\left(C_{F}\left[\frac{g_{F R}}{L}\right]\right)(\text { Yield })}$

873 where $R R$ is the recycle ratio of solvent. 
The equipment cost was calculated according to Eq. (B.4).

$875 E C\left[\frac{\$}{k g_{F R}}\right]=\frac{(\text { SMB price })}{(\text { Depreciation Time })(\text { Utilization Factor })(\text { Production })}=\frac{(3,000,000[\$])}{(7[\text { years }])(.96)\left(10,000,000\left[\frac{\mathrm{kg}_{F R}}{\text { year }]}\right)\right.}$

876 Appendix C - Cost Optimization Algorithm

The cost optimization algorithm that was used to determine the optimum yields, particle

878 radius, column configuration, column length, zone velocities, and port velocity is shown in Fig.

879 C.1. Material properties (other than particle radius), dead volume, pressure limit, feed

880 concentration, packing density, fluid viscosity, and production scale were held constant. The

881 total number of columns was varied from eight to twelve and each zone was constrained to have

882 at least two columns. The number of columns in Zone IV was calculated from the column

883 balance of the other zones and the total number of columns. $N_{D}{ }^{*}$ ranged from 0.1 to $20, P_{e b}{ }^{*}$

884 ranged from 5 to $1,000, R_{p}$ ranged from $112 \mu \mathrm{m}$ to $450 \mu \mathrm{m}$, and yields ranged from 0.990 to

885 0.999. The initial values of the variables were passed to the SSWD algorithm, Fig. C.1b, which

886 used an initial guess for $\beta_{i}^{j}$ and $\Gamma^{j}$ and then evaluated $u_{0}^{j} / v$. The SSWD algorithm then calculated

$887 \beta_{i}^{j}$ (Eq. (A.3)), and $\Gamma^{j}$ (Eq. (A.5)) from $u_{0}^{j} / v$. If the calculated values were not within 0.001 of

888 the guessed values, then the $u_{0}^{j} / v$ calculations were repeated with the calculated values of $\beta_{i}^{j}$ and

$889 \Gamma^{j}$. Once the values were within the tolerance, $L_{c}$ was calculated from $P_{e b}{ }^{*}$ using Eq. (A.13) and

$890 v$ was calculated from $N_{D}{ }^{*}$ using Eq. (2). Once $v$ was calculated, the zone velocities were

891 calculated using Eq. (4). The solvent consumption and sorbent productivity were then calculated

892 using Eq. (A.10a) and Eq. (A.12a), respectively. The overall separation cost (total cost) was then

893 calculated using Eqs. (B.1-B.4). The pressure drop of the system was calculated using Eq. (4)

894 and compared to the set pressure limit of 100 psi per zone. If the calculated pressure exceeded

895 the pressure limit, then the design was not considered feasible. After the pressure check, the

896 Peclet number was increased and the process repeated until $P_{e b}{ }^{*}=1,000$. Then, $N_{D}{ }^{*}$ was 
897 increased and the $P_{e b}{ }^{*}$ cycle repeated. Once $N_{D}{ }^{*}$ reached a value of 20 , the particle size increased 898 and the $N_{D}{ }^{*}$ and $P_{e b}{ }^{*}$ cycles repeated. In similar fashions, all the cycles were repeated for varying 899 column configuration and yield of each component. After all the cycles were completed, the

900 minimum cost was found from all the stored values and the associated cost, $Y_{i}, R_{p}, L_{c}, N^{j}, u_{0}^{j}$, and $901 \quad v$ were determined. This algorithm was implemented using MATLAB R2014a on a laptop 902 computer with a total run time of less than one minute.

903 


\section{Figure Captions}

905 Figure 1. Overview of Purdue process to recover high-purity polycarbonates and FRs from an electronic waste stream. SEPoR process and cost estimate described in Weeden et al. [15]. ${ }^{\dagger}$ Cost estimate for 10,000 tonnes FR/year, Table 7.

Figure 2. Diagram of a four-zone SMB. (a) Step N; (b) step N + 1. Large red spheres represent the fast component; small green spheres represent the slow component.

Figure 3. Standing Wave Design end-step concentration profiles for (a) binary, (b) ternary - Split 1, and (c) ternary, Split 2 non-ideal separations. Pump configuration for the SMB experiments is also shown. The feed, desorbent, extract, and Zone IV recycle flowrates were controlled by individual pumps while the rest of the streams were determined by mass balance. The black arrows indicate the standing waves in each of the four zones. Asterisks indicate pinched waves.

Figure 4. SSWD overview for ternary SEC-SMB.

Figure 5. (a) Blue dextran pulse to determine bed void fraction, and (b) component frontals to determine size-exclusion factors and diffusivities of each component. BPADP frontal not shown as it overlaps with the RDP data.

Figure 6. (a) SMB Run 1 preloaded column profiles predicted by VERSE, (b) column profiles for $K_{s e, S A N}=0.63$ (c) column profiles for $K_{s e, S A N}=0.61$ (top), extract history (bottom left), and raffinate history (bottom right) for SMB Run 1, end of step 33. Lines are from VERSE simulations while squares and diamonds are HPLC data points. Design in Tables 5, 6.

Figure 7. (a) column profiles for Run 2 at the end of the $51^{\text {st }}$ step. VERSE simulations used a column length of $65 \mathrm{~cm}$. (b) column profiles (top), extract history (bottom left), and raffinate history (bottom right) for SMB Run 2 using a column length of $63 \mathrm{~cm}$, end of step 51. Lines are from VERSE simulations while squares and diamonds are HPLC data points. Design in Tables 5, 6.

Figure 8. Column profiles (top), extract history (bottom left), and raffinate history (bottom right) for SMB Run 3, end of step 56. Lines are from VERSE simulations while squares and diamonds are HPLC data points. Design in Tables 5, 6.

Figure 9. (a) Preloaded column profiles obtained from VERSE simulations, (b) column profiles (top), extract history (bottom left), and raffinate history (bottom right) for SMB Run 4, end of step 27. Lines are from VERSE simulations while squares and diamonds are HPLC data points. Design in Tables 5, 6.

Figure 10. Comparison of pre-loading strategies and regular startup for binary SEC-SMB. SMB design is the same as Run 4. Effluent histories: (a) raffinate and (b) extract. RDP concentrations not shown in (a) since all strategies resulted in very low concentrations of RDP throughout the simulations. Likewise the SAN concentrations are not shown in (b) except for the feed loading strategy which has significant SAN contamination. 
943 Figure 11. (a) Extract and (b) raffinate histories from SMB Run 5. No column profiles were 944 taken for Run 5. Design in Tables 5, 6.

945 Figure 12. (a) SMB Run 6 preloaded column profiles predicted by VERSE, prior to start of SMB 946 operation; (b) SMB column profile at start of step 8; (c) start of step 16; (d) start of step 24; (e) 947 start of step 32; (f) start of step 40; (g) column profiles (top), extract history (bottom left), and 948 raffinate history (bottom right) for SMB Run 6, end of step 40. Lines are from VERSE

949 simulations while squares, triangles, and diamonds are HPLC data points. Design in Tables 5, 6.

950 Figure 13. (a) Inverse of total cost surface for ternary separation with pressure limit surface and 951 (b) contour plot of (a) with pressure limit (black line) and minimum cost point (x). $N^{j}=2-2-2-2$, $952 D V=1.9 \%, \Delta P_{\max }=100$ psi per zone, $Y_{i}=99 \%, R_{p}=112 \mu \mathrm{m}$.

954 Figure 14. Total, sorbent, solvent, and equipment costs versus $N_{D}{ }^{*}$ at $P_{e b}{ }^{*}$ for minimum cost. 955 Vertical black line represents the pressure limit - operating at $N_{D}{ }^{*}$ values to the right of the line 956 will satisfy the pressure requirement.

958 Figure C.1. (a) algorithm for optimizing 14 decision variables (yields, particle size, column 959 configuration, column length, zone velocities, and port velocity) for minimum cost and (b) 960 algorithm used by SSWD to determine column length, $\beta_{i}^{j}, \Gamma^{j}$, zone velocities, and port velocity 961 from given dimensionless groups.

962 
Figure 1

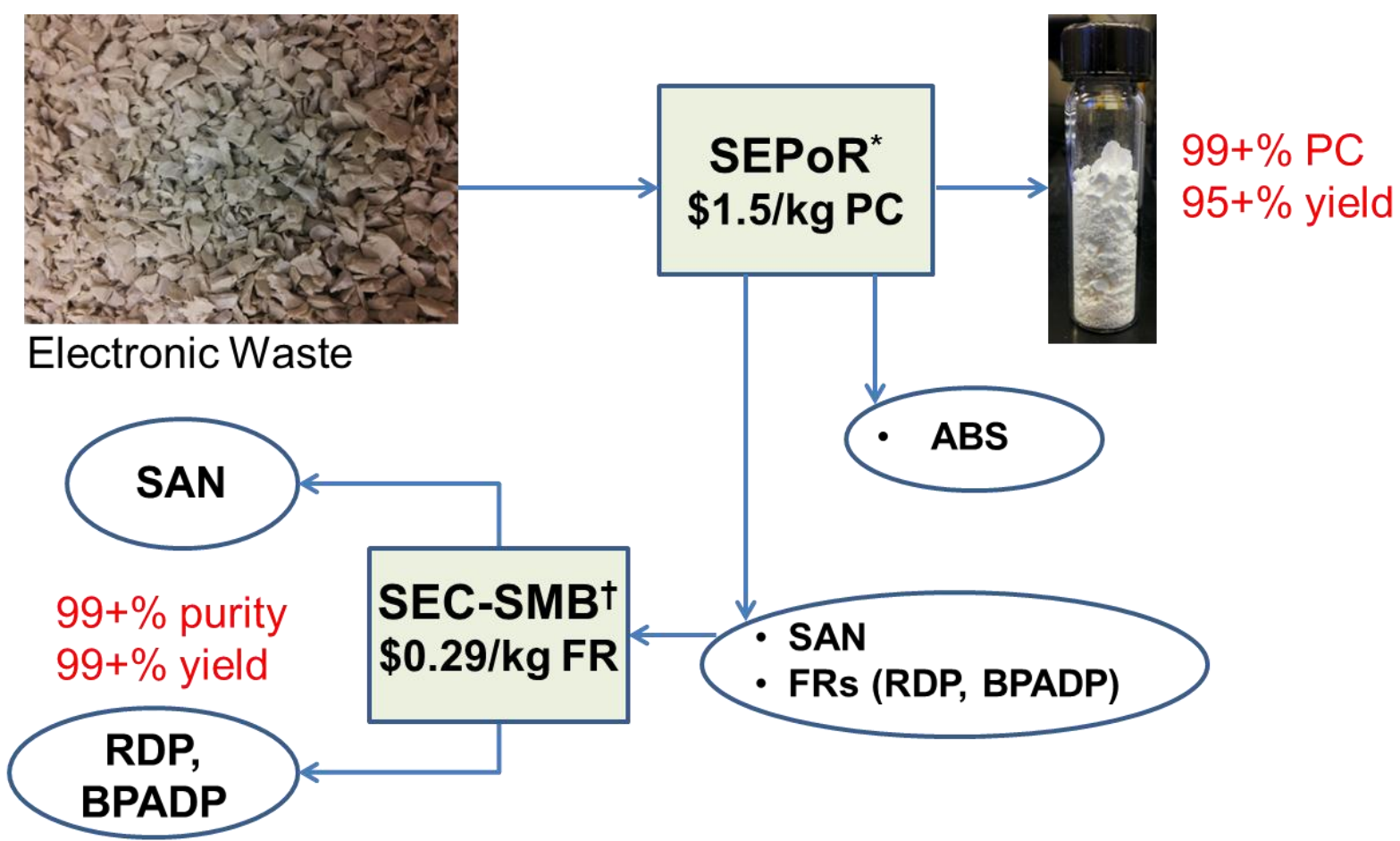


(a)

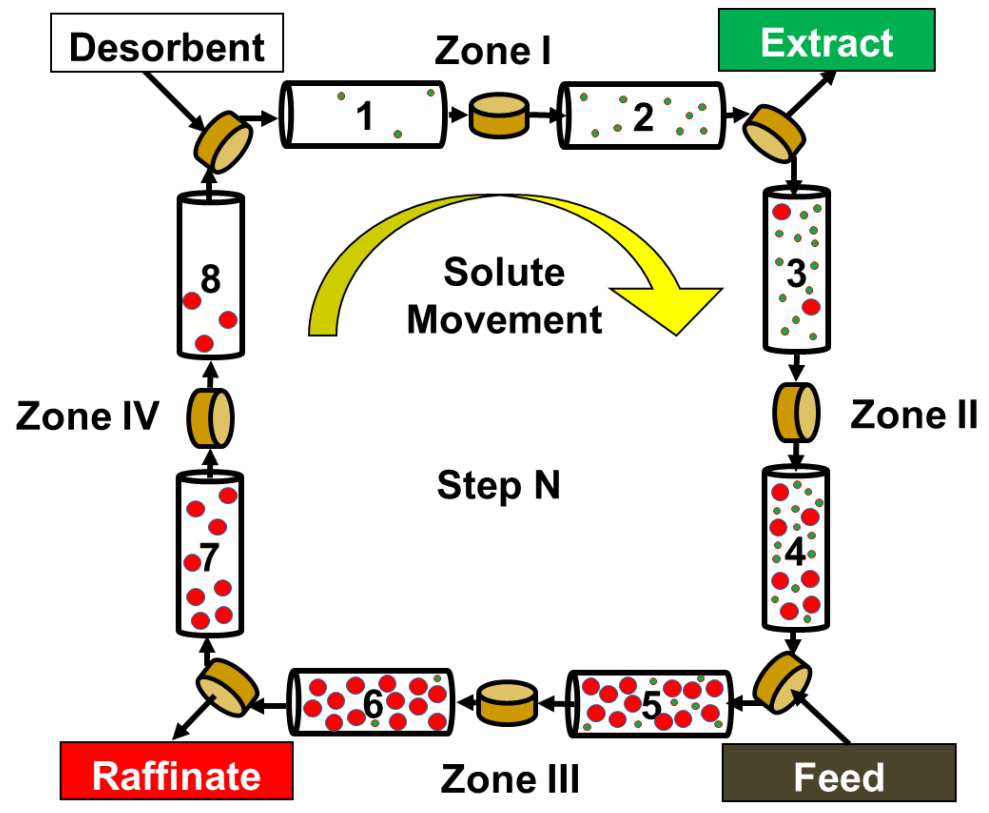

(b)

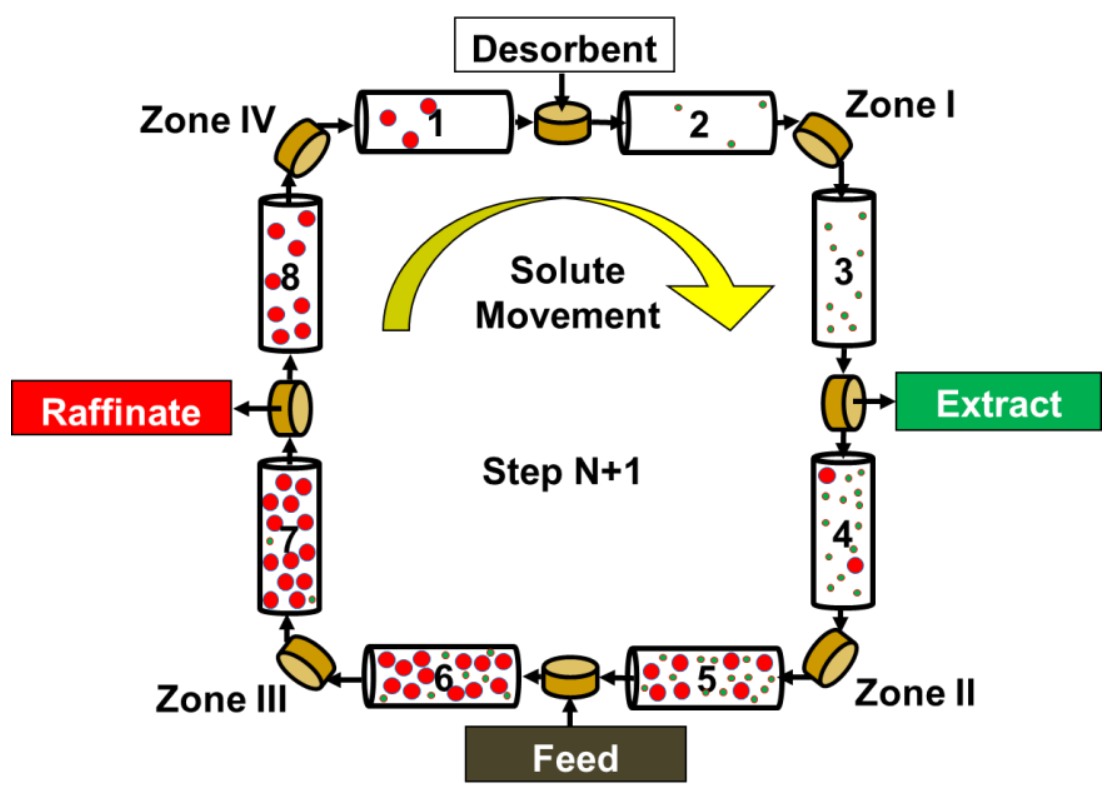


(a)

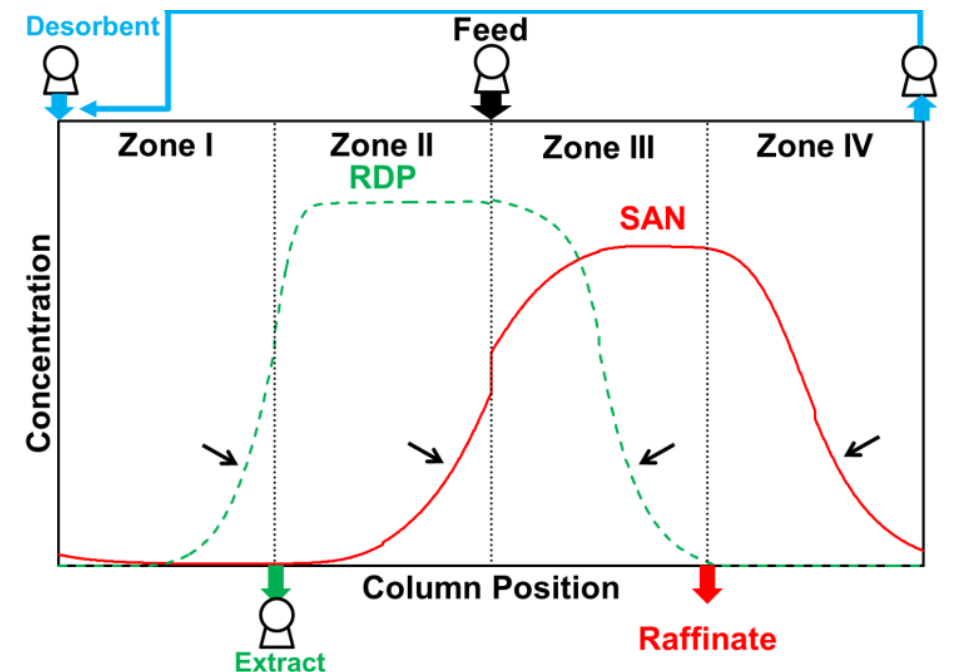

(b)

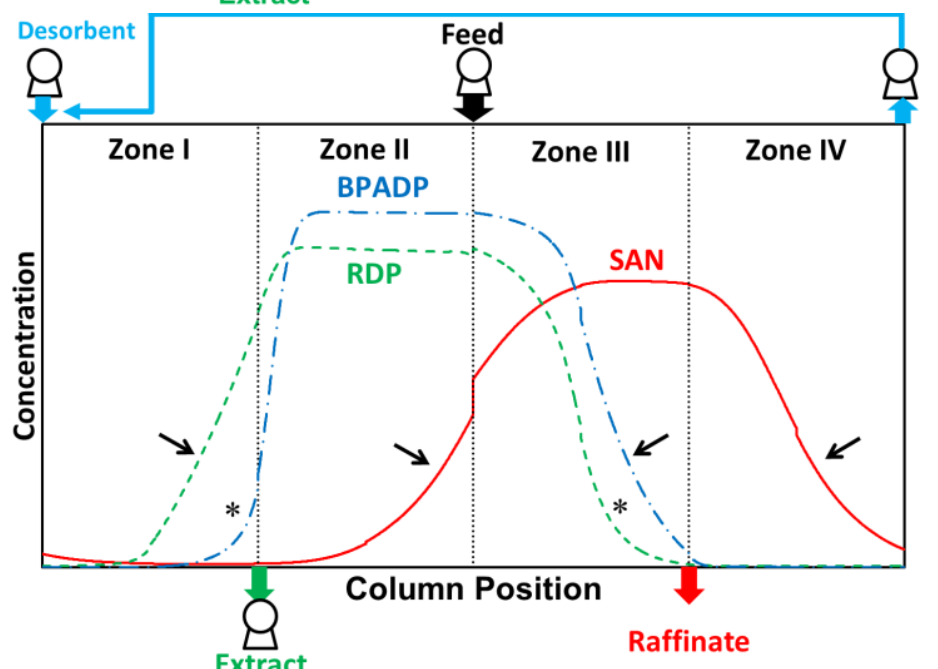

(c)

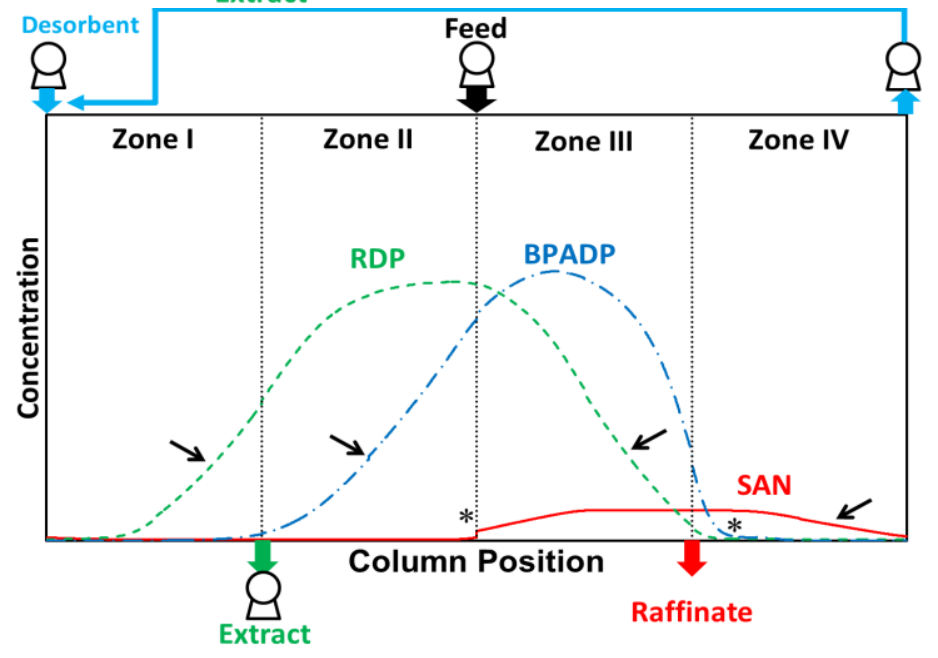




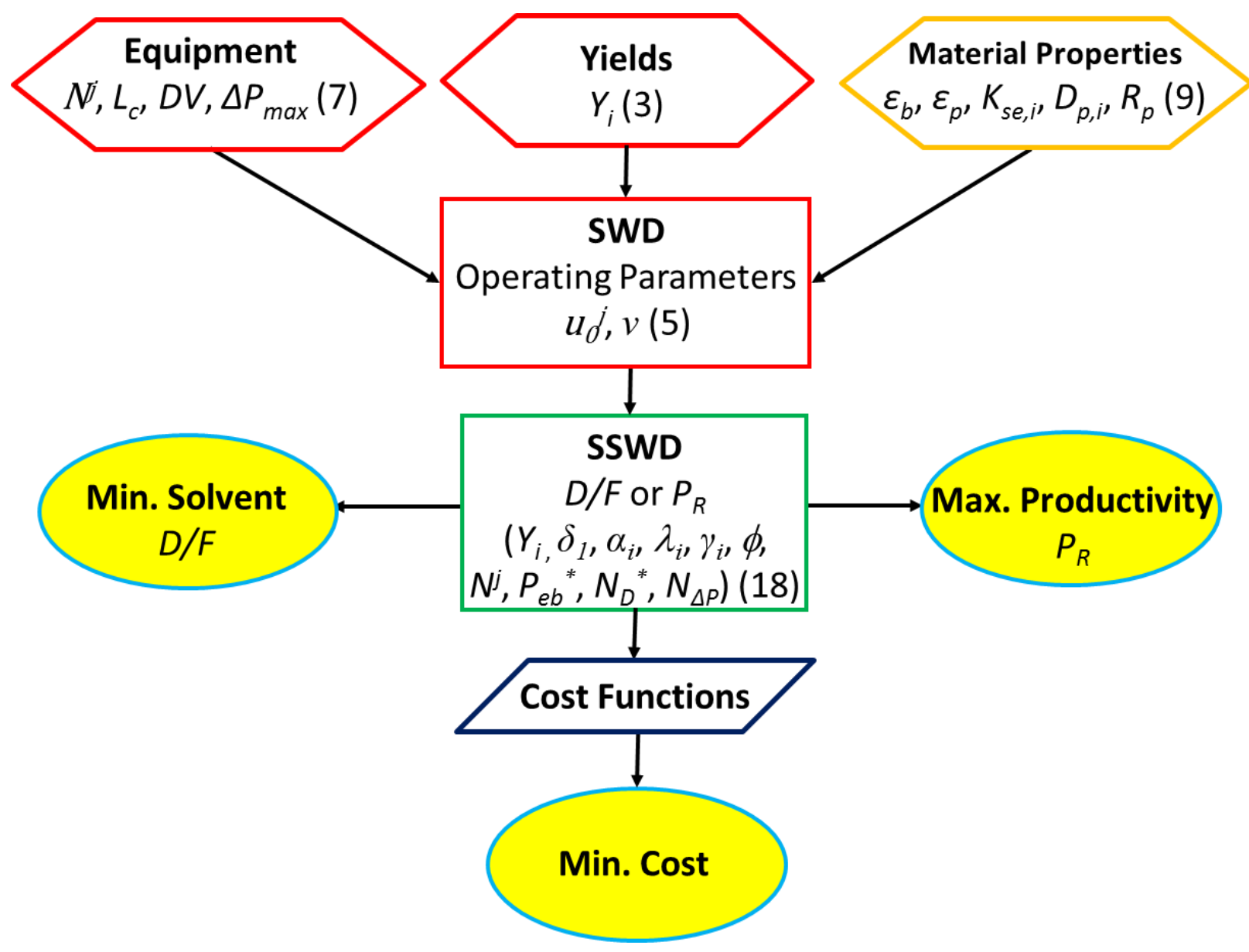


Figure 5

(a)

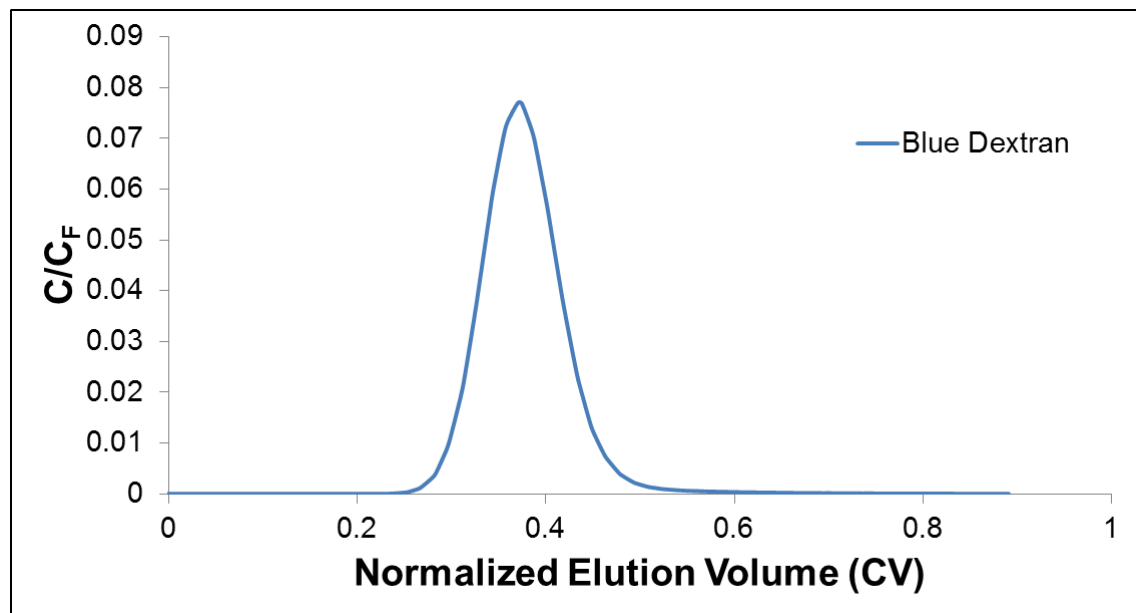

(b)

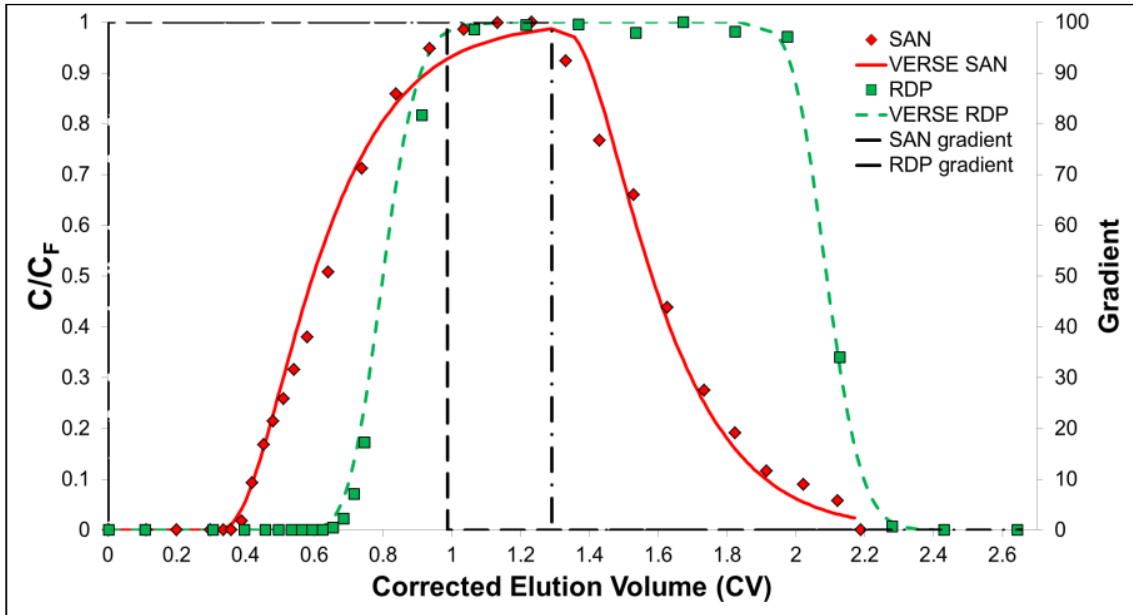


(a)

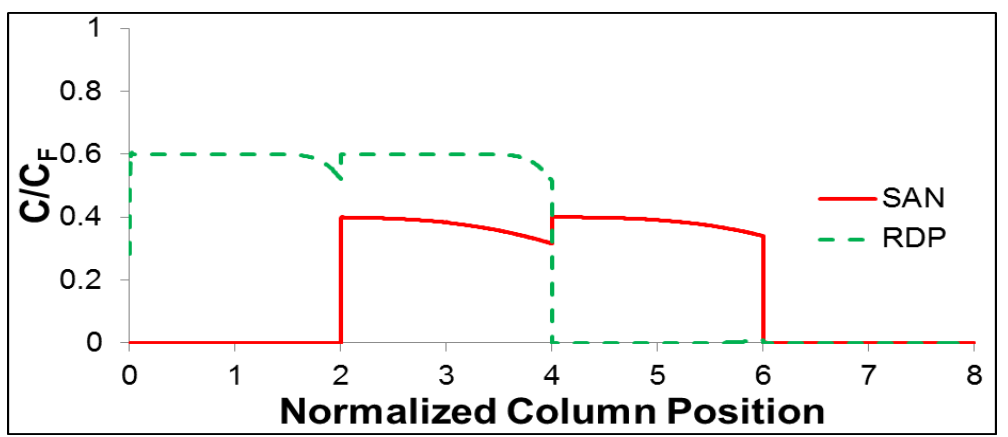

(b)

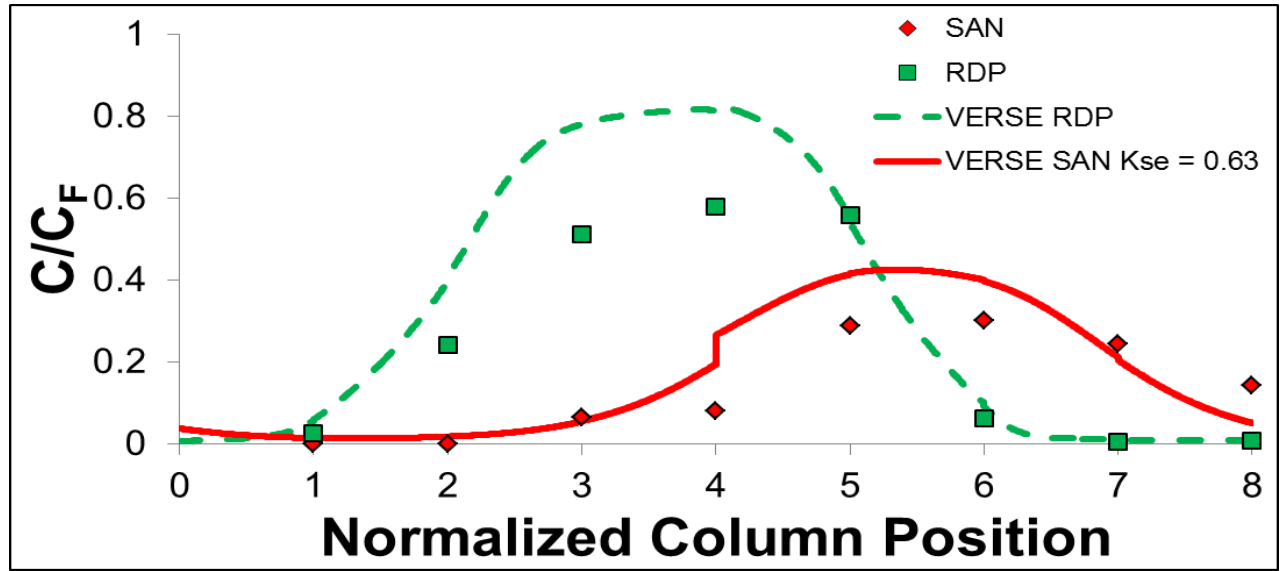

(c)
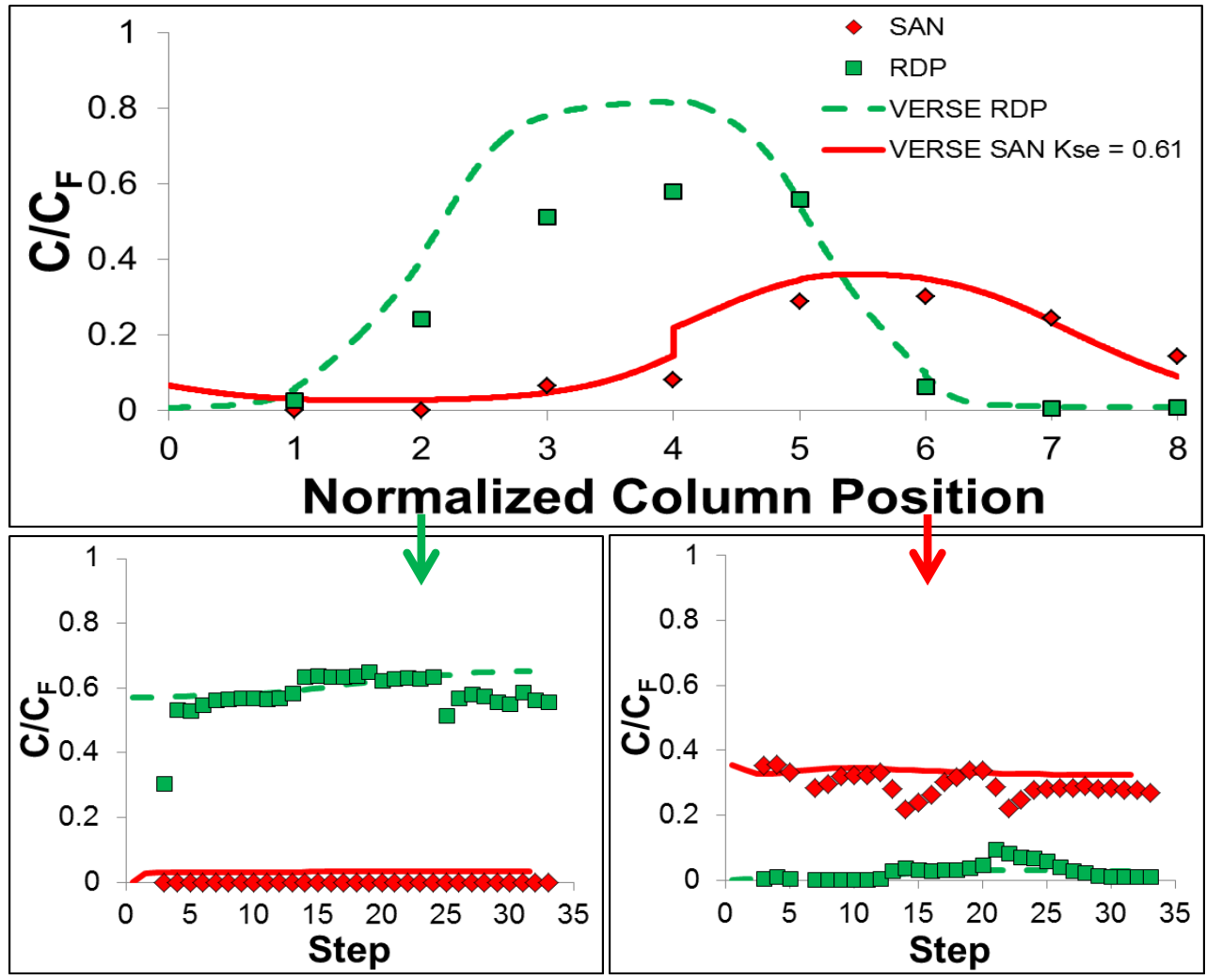
(a)

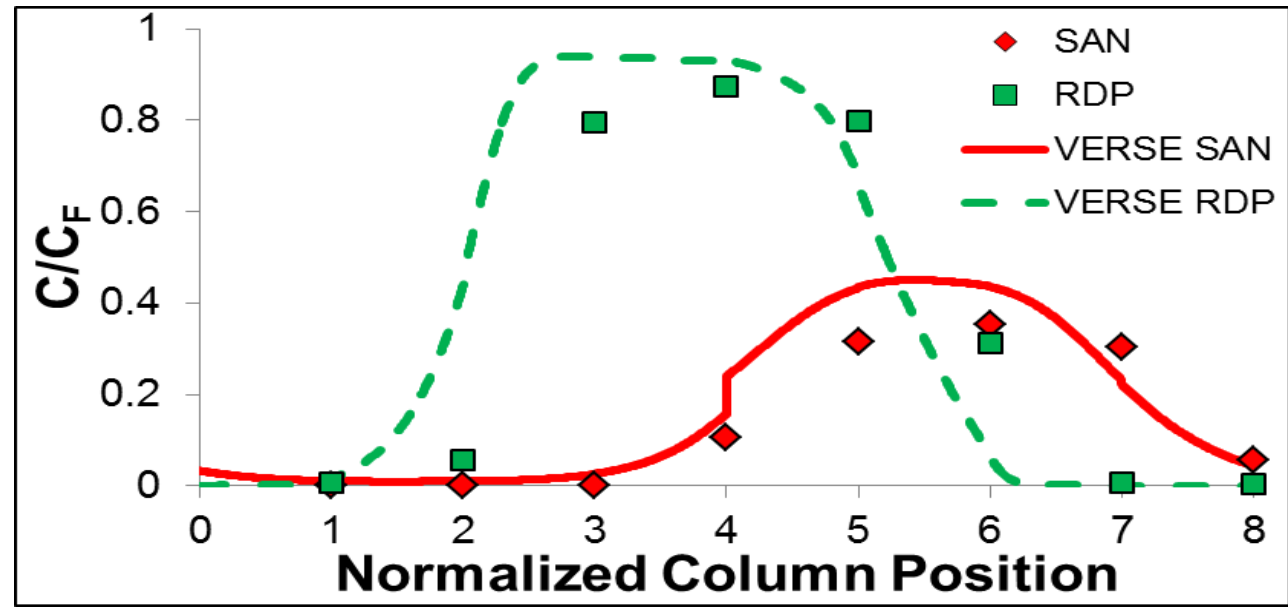

(b)
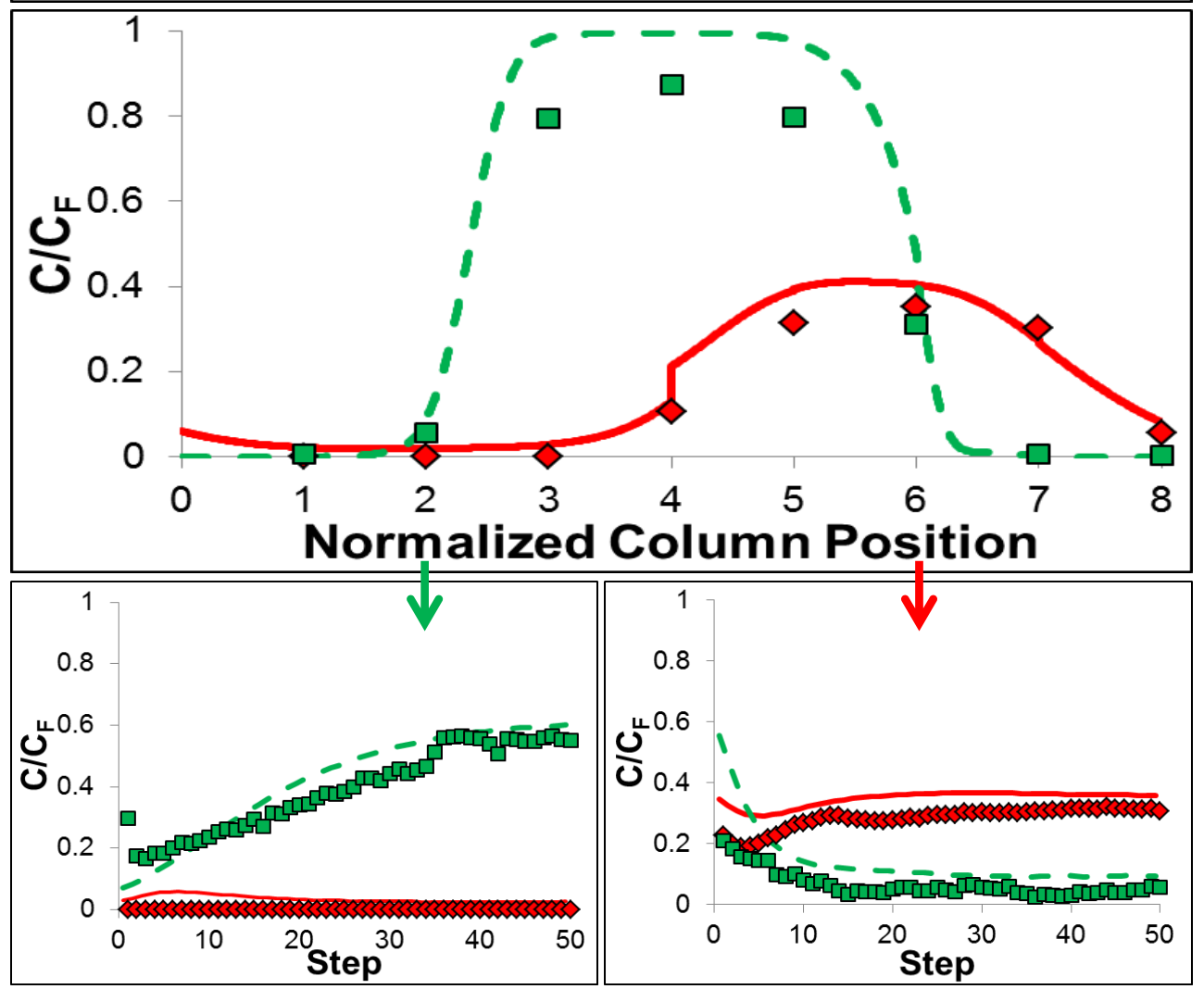


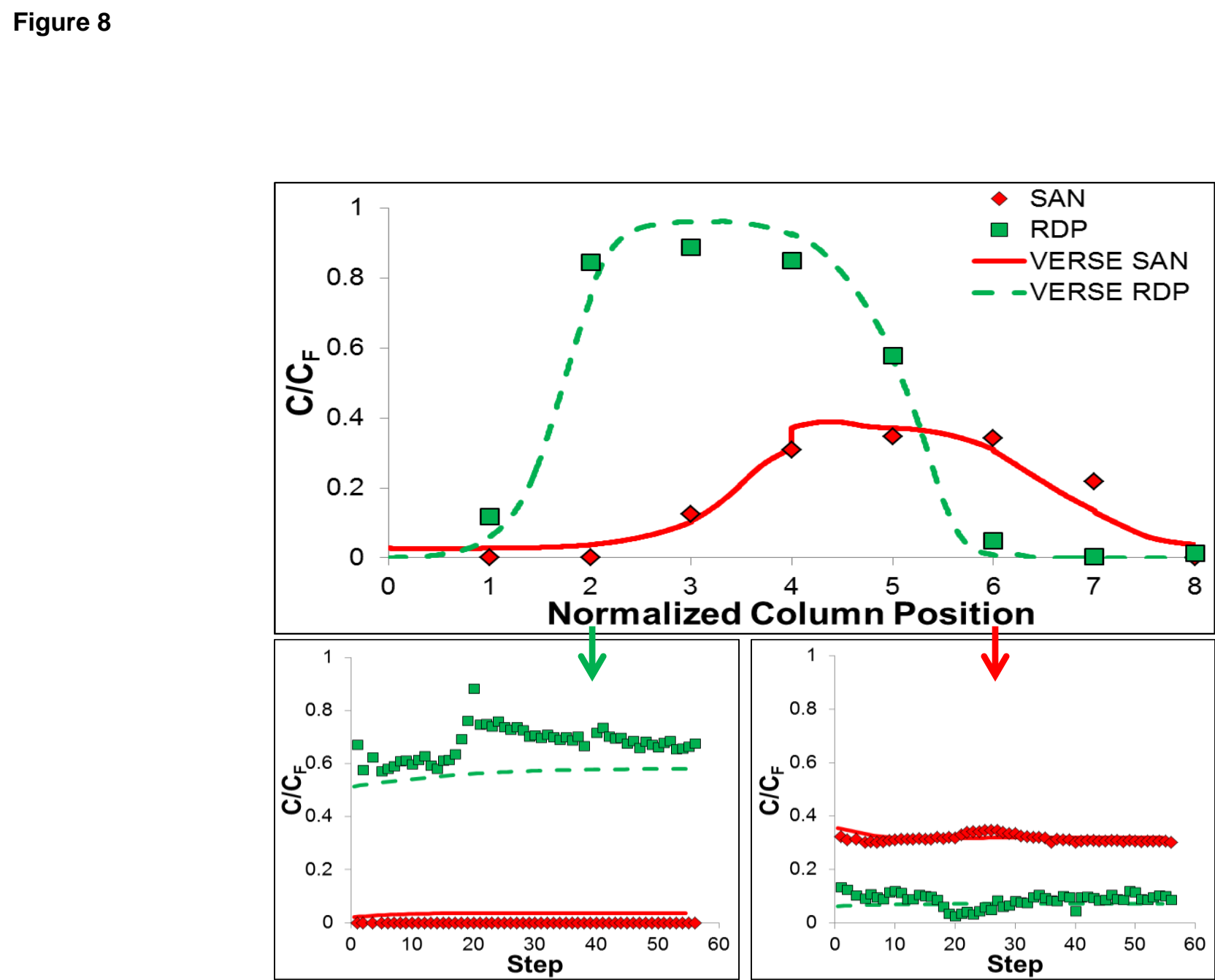
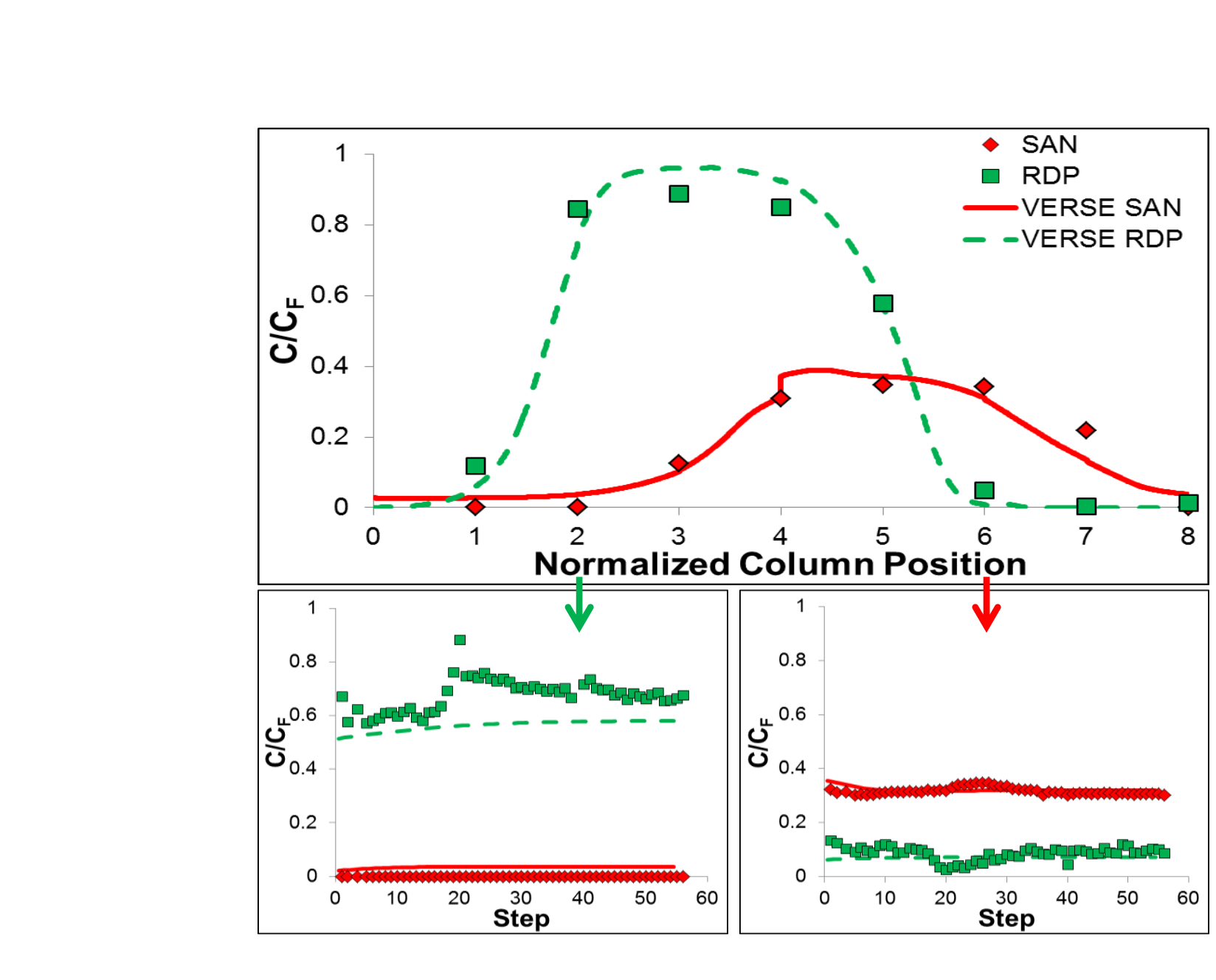

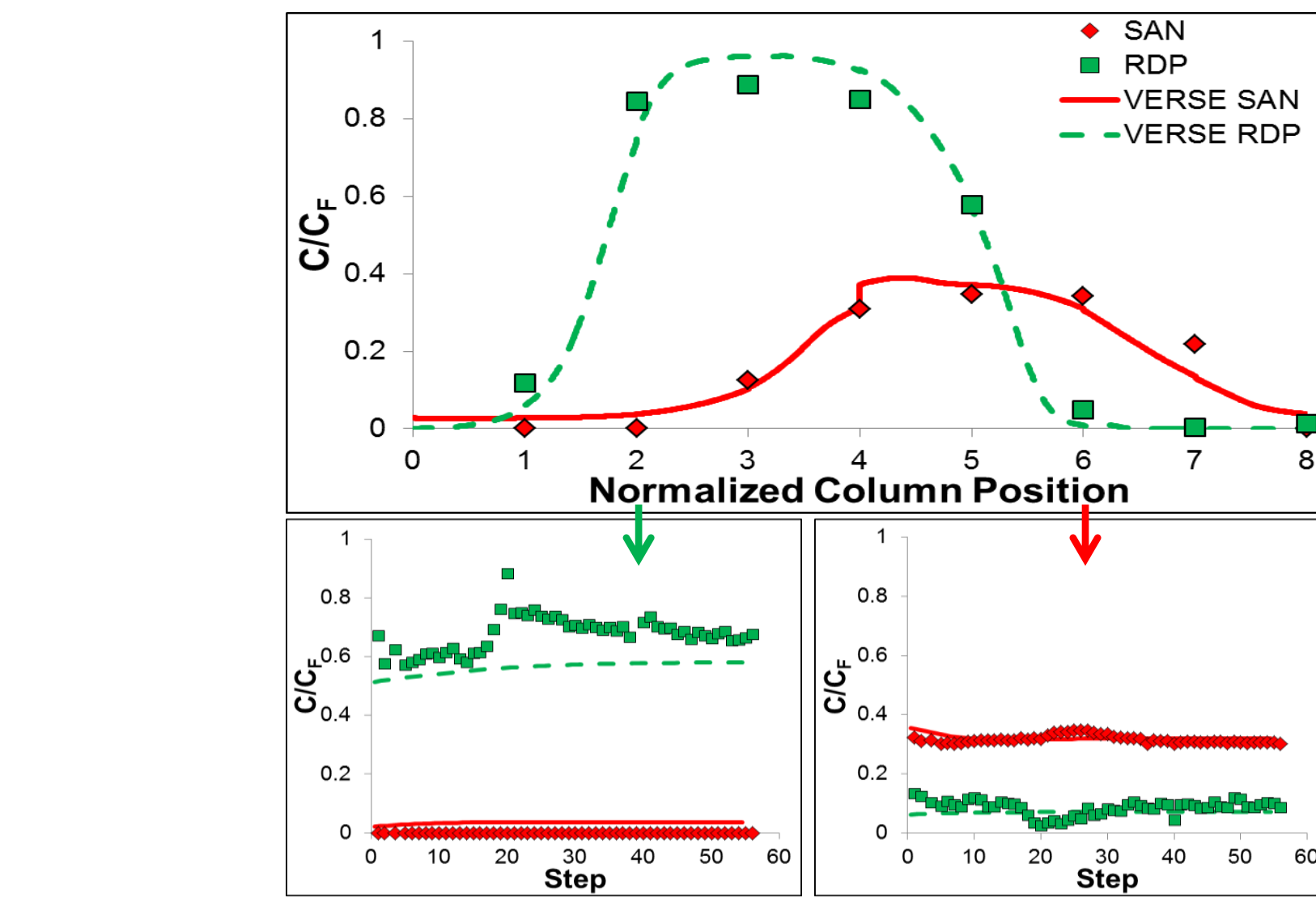
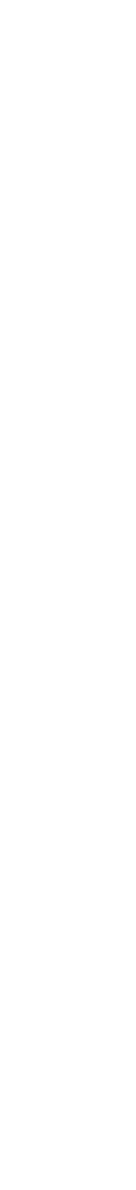
(a)

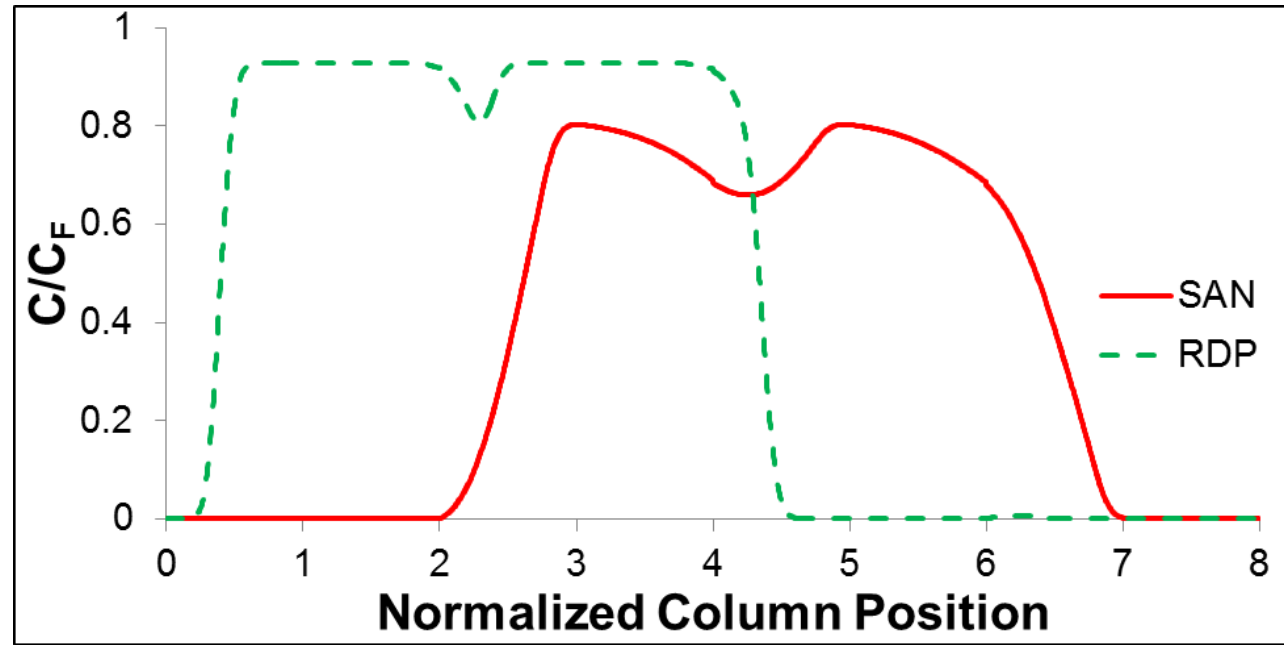

(b)
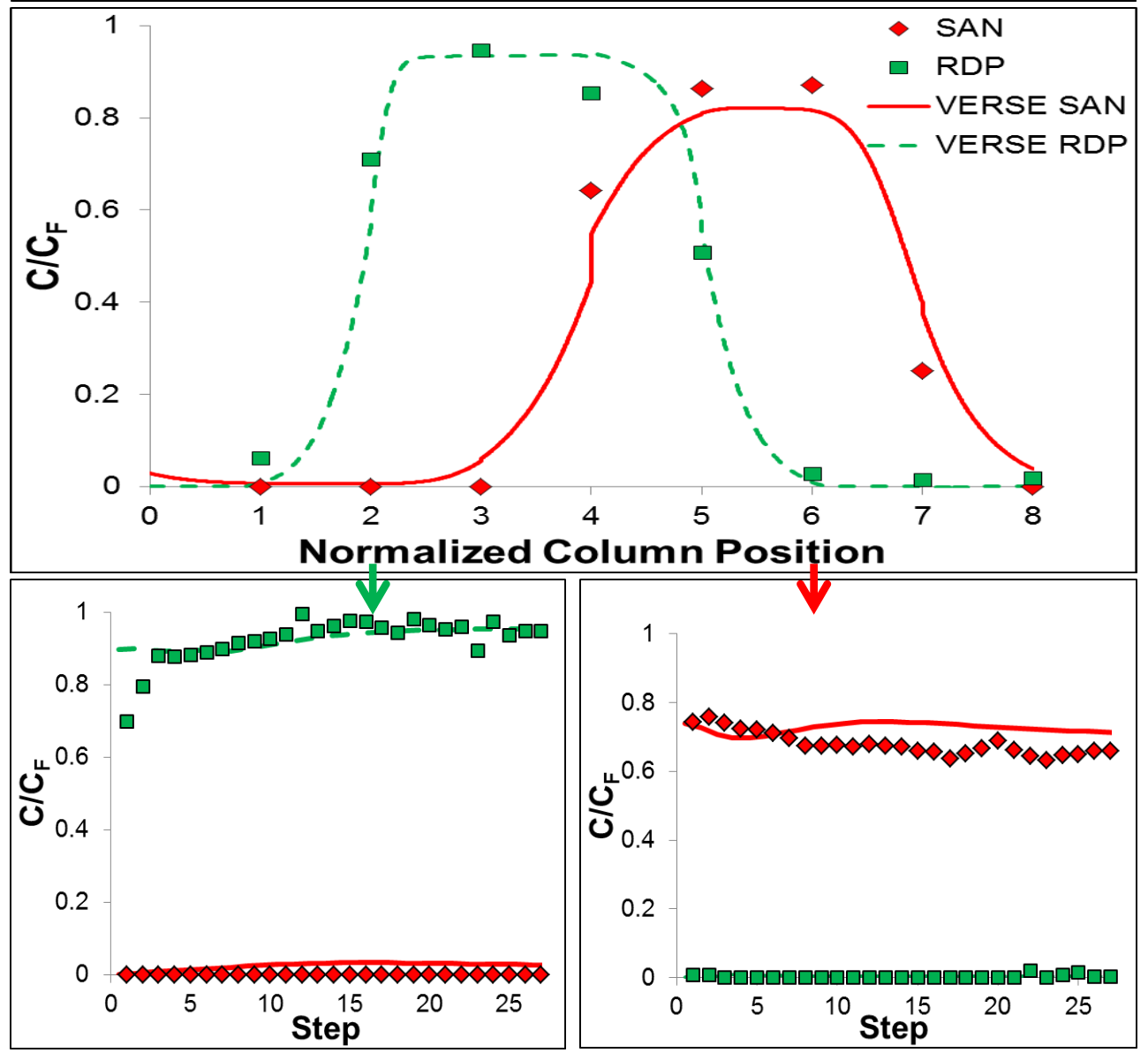
(a)

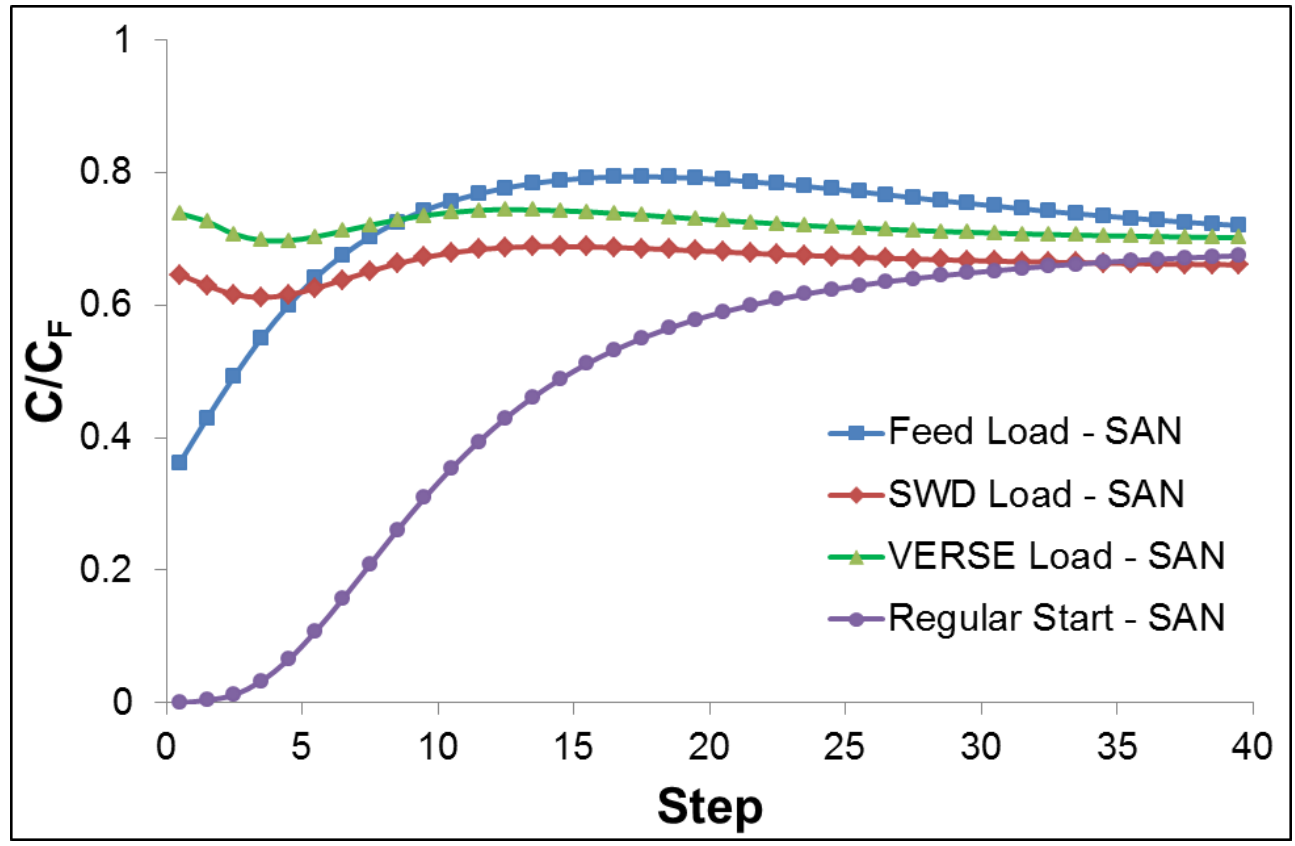

(b)

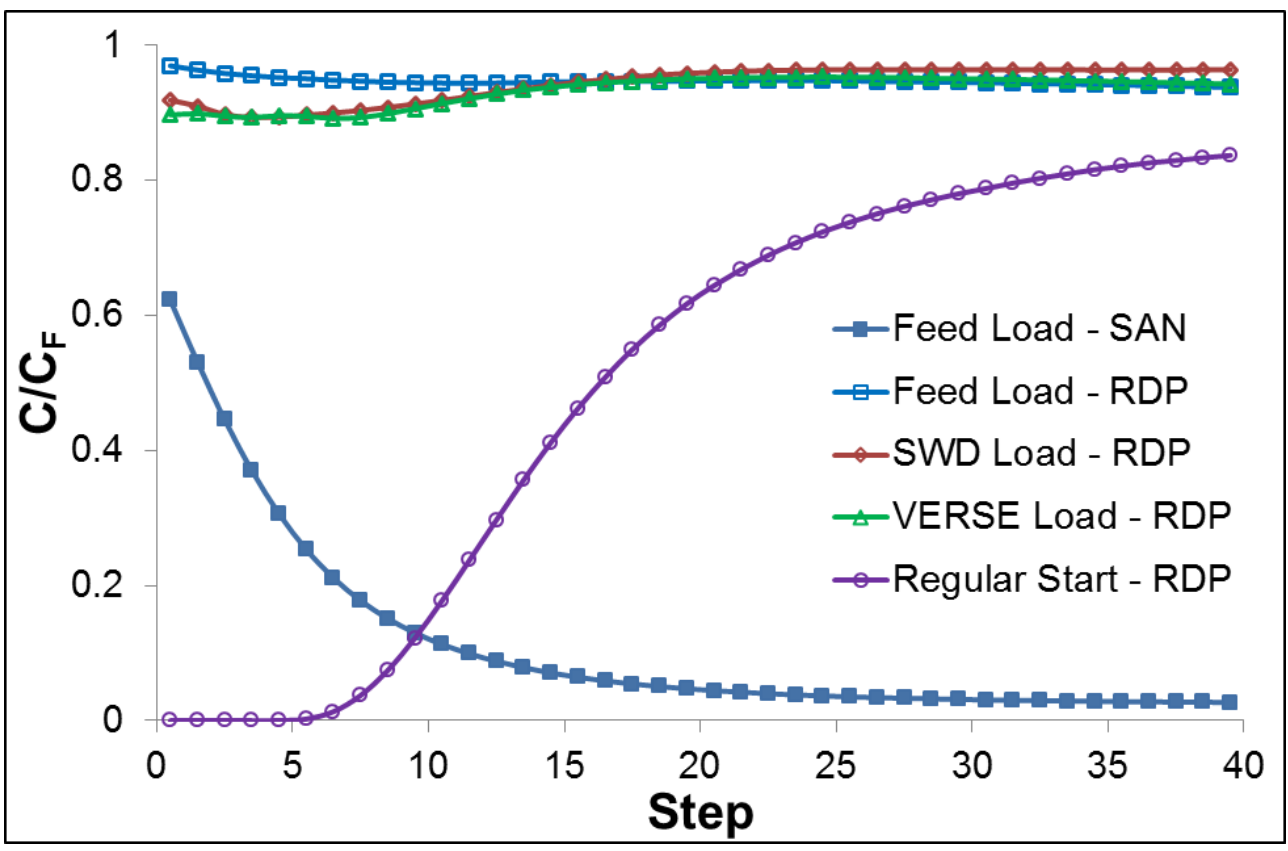


(a)

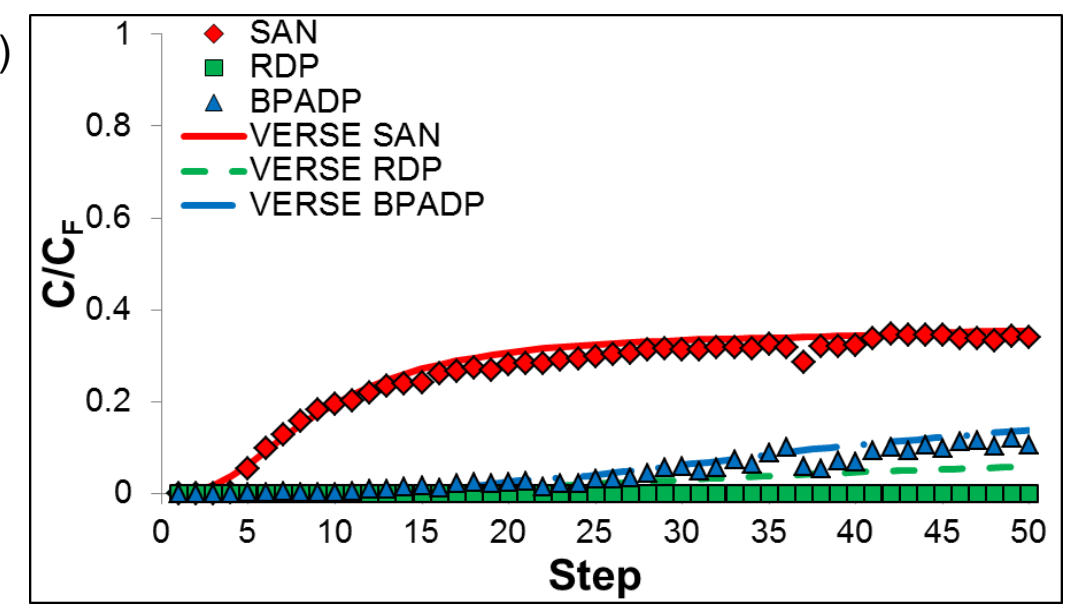

(b)

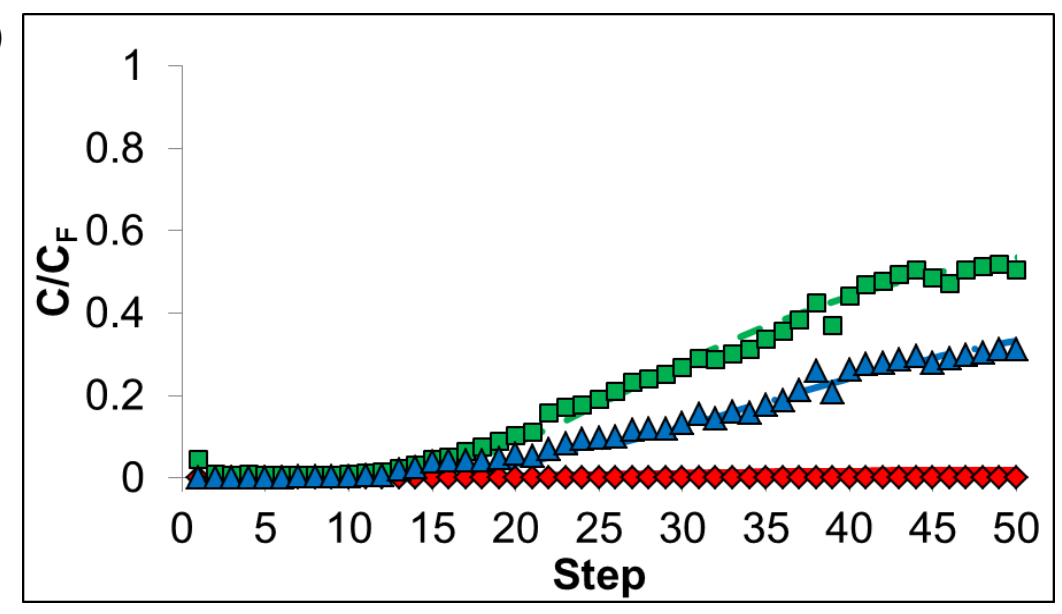

$$
\text { . }
$$

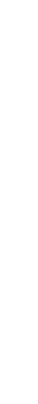

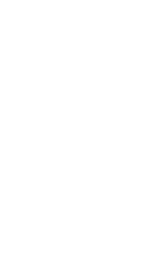
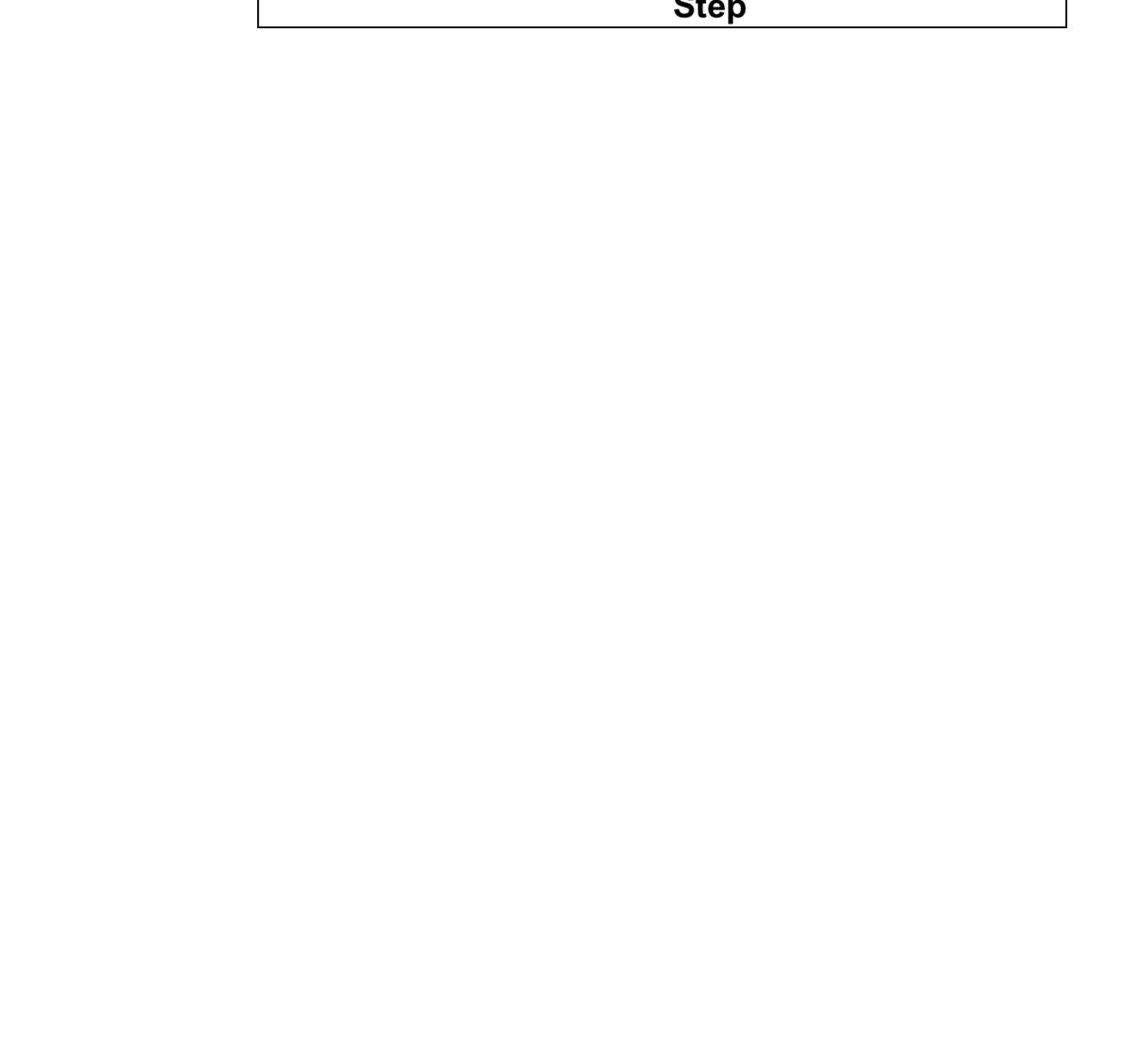
(a)

(b)
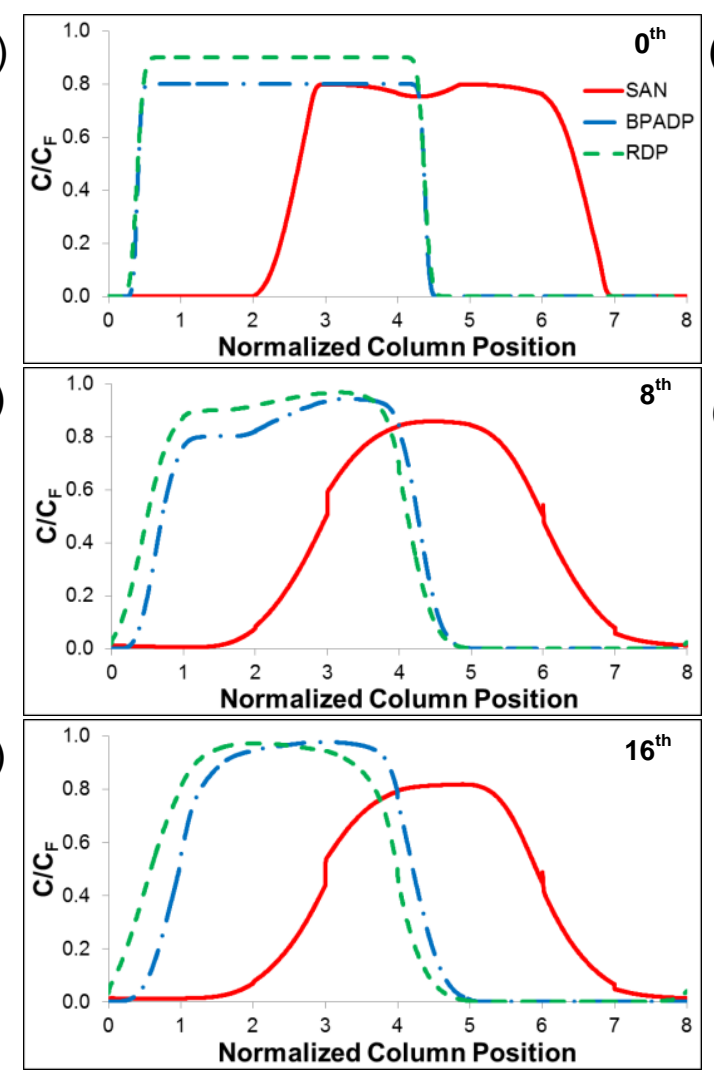

(d)

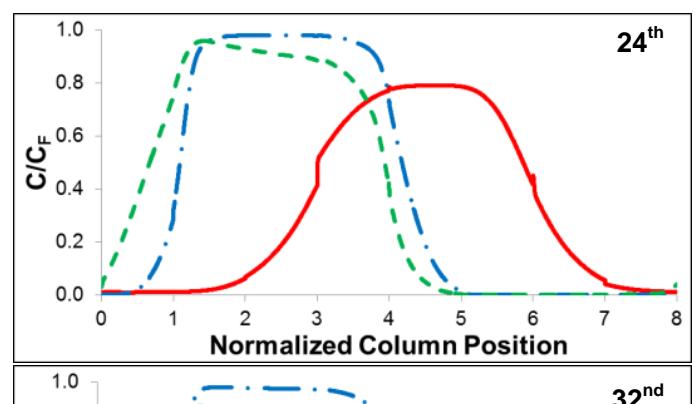

(e)

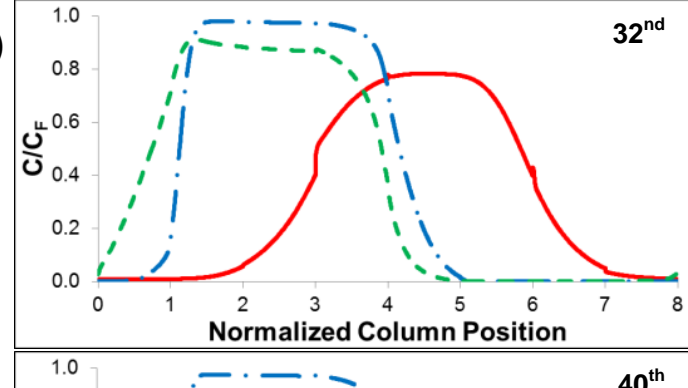

(f)

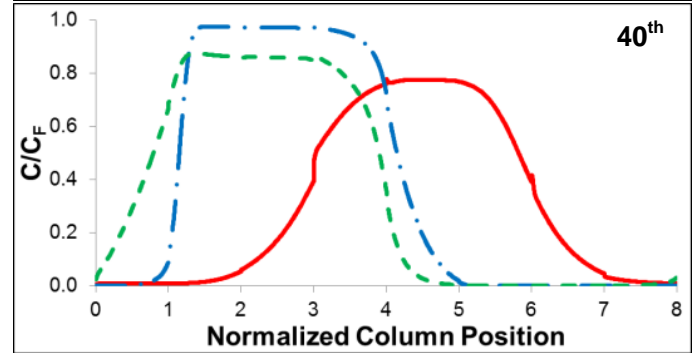

(g)

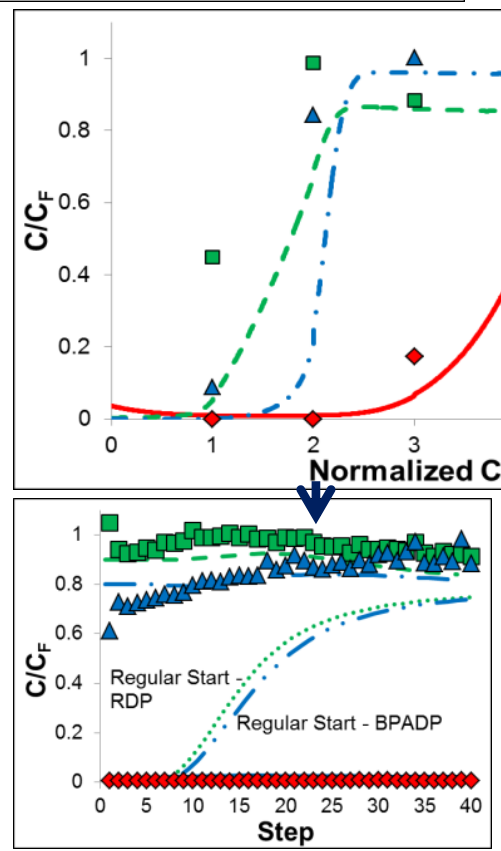

- SAN

RDP

- VERSE SAN

- -VERSE RDP

- VERSE BPADP

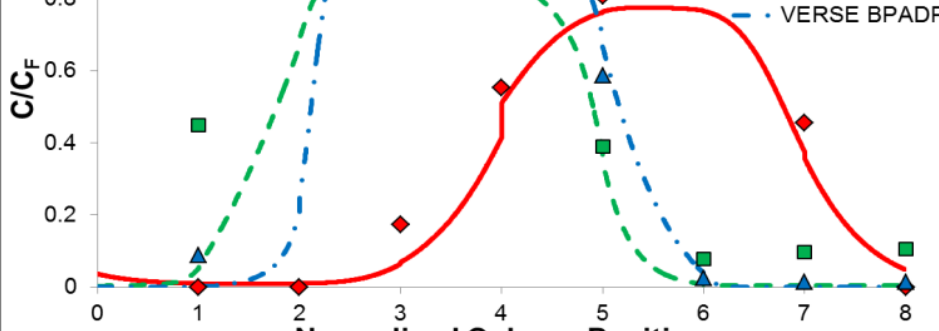

olumn Position

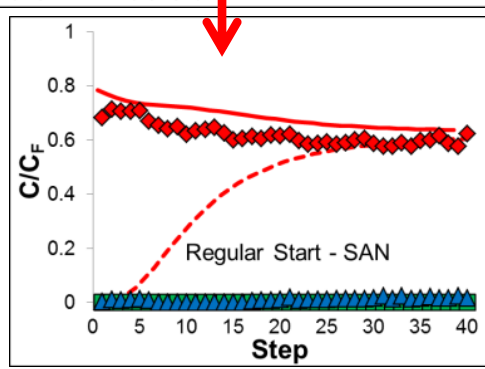


(a)

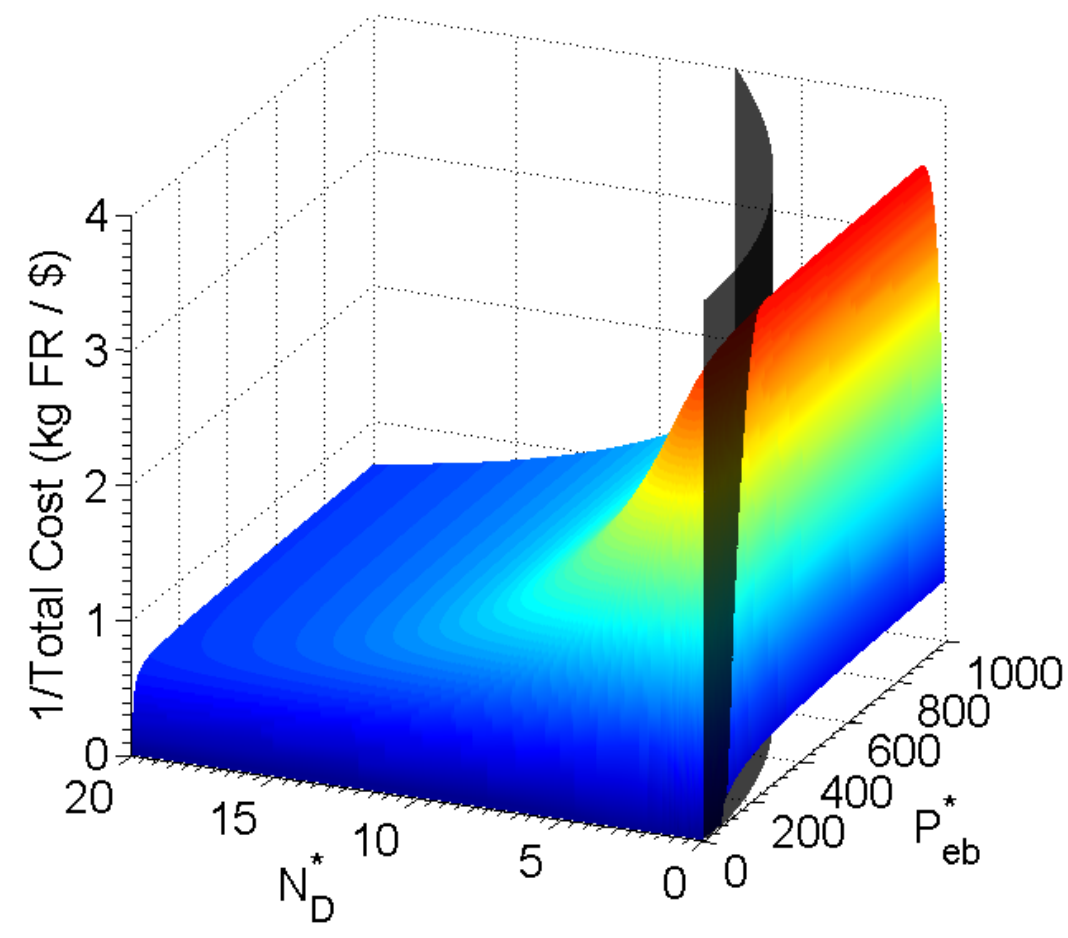

(b)

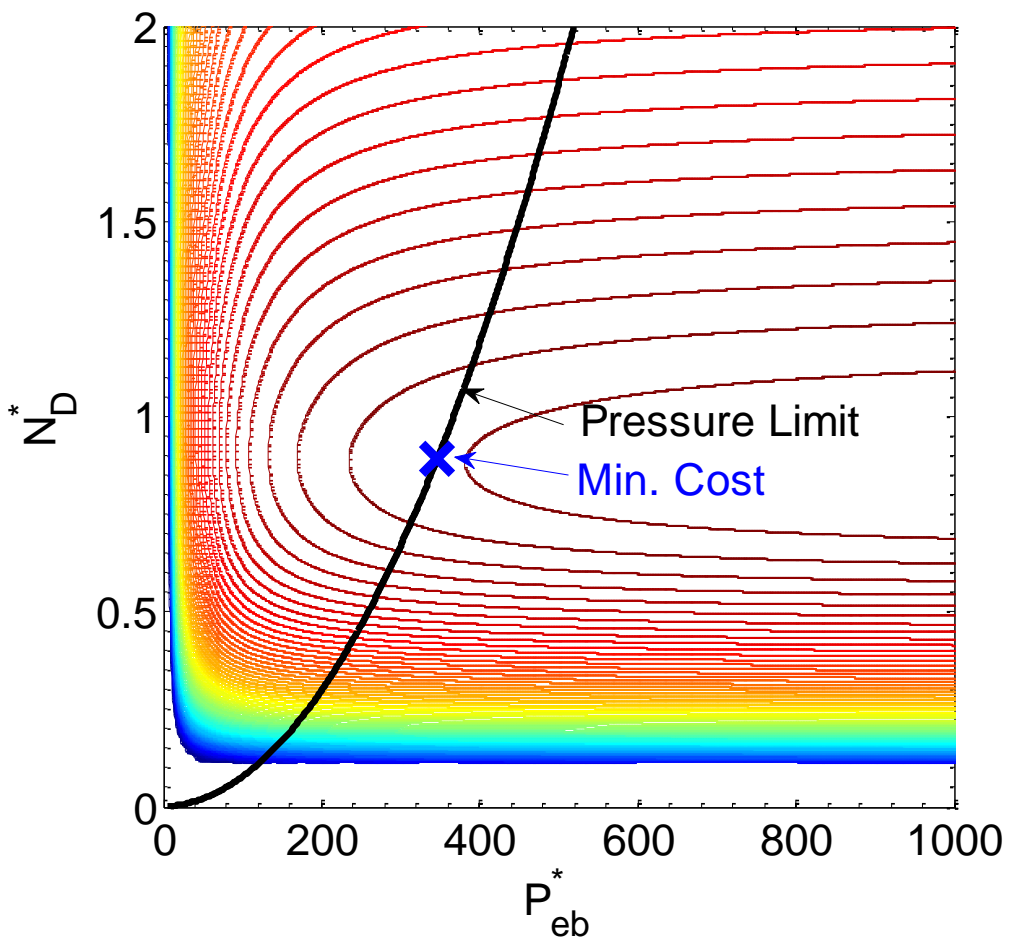




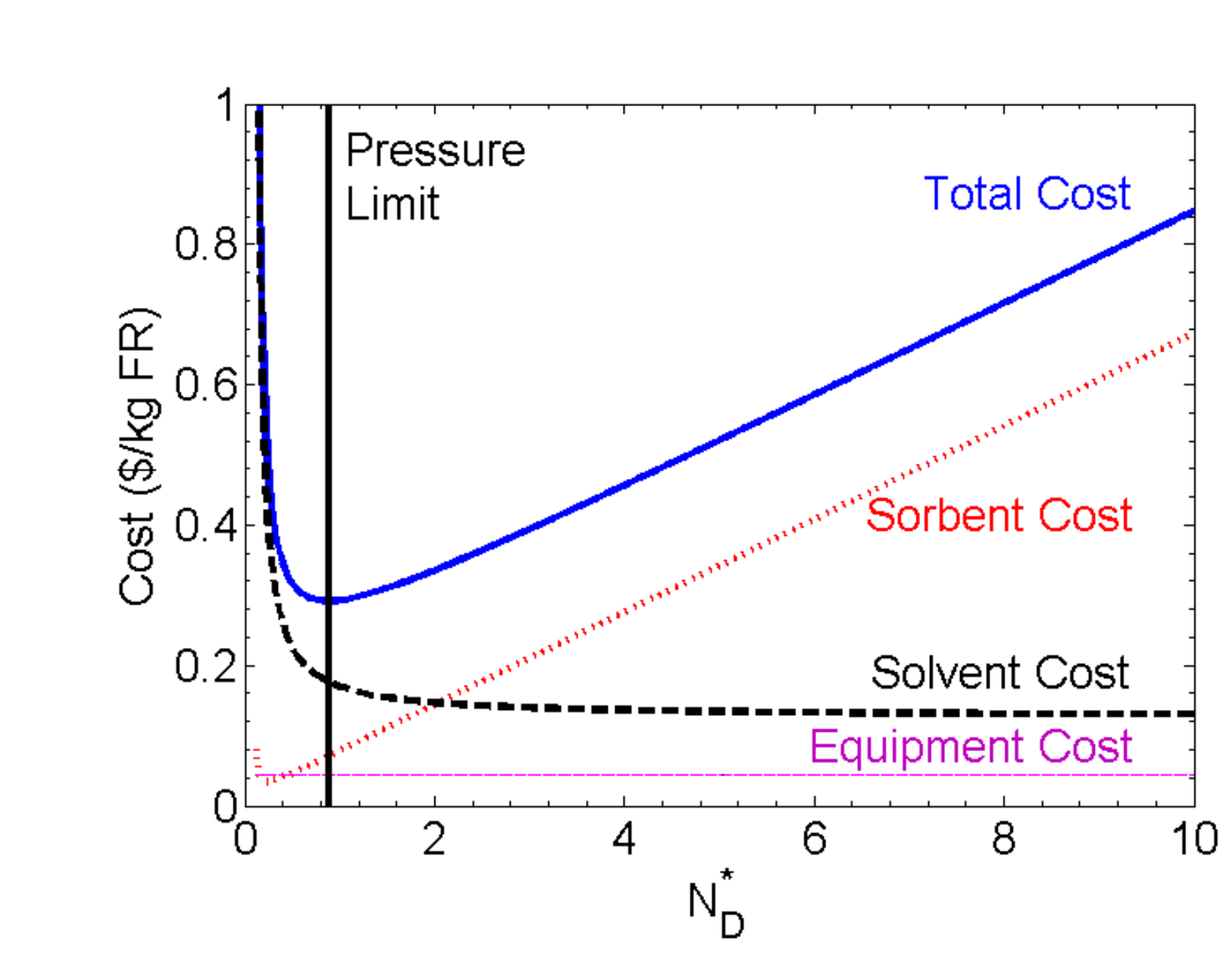

Figure 14

.

\section{.}

.
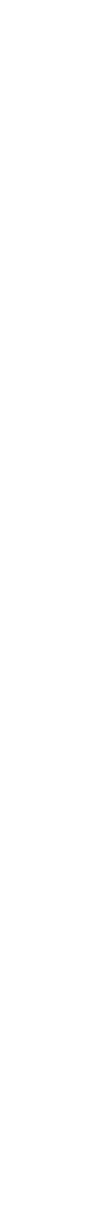

Figure 14

.

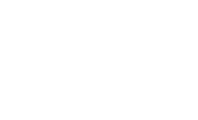




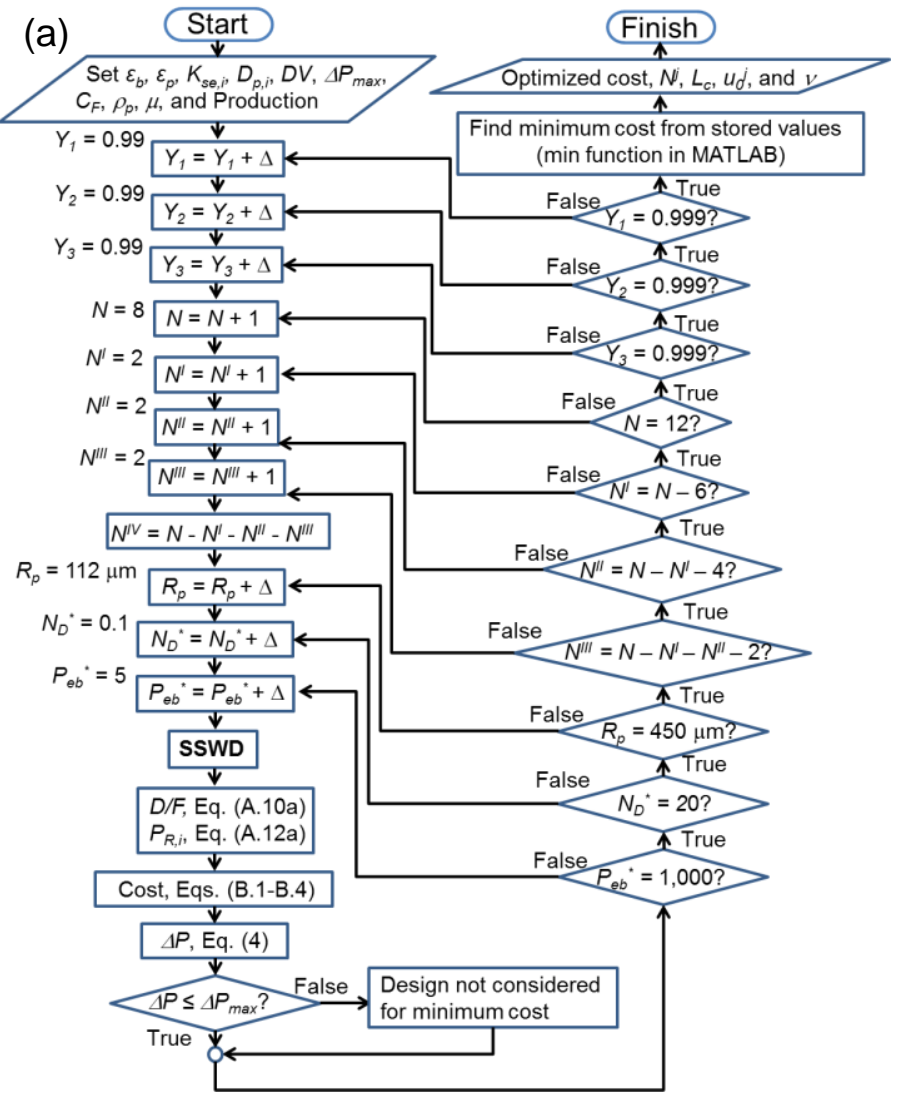

(b)
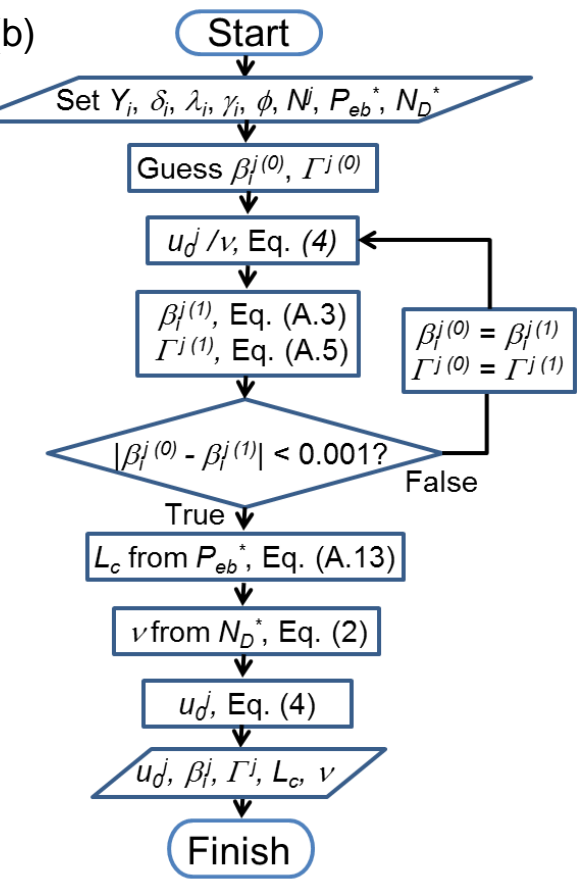
Table 1

Main components of one of the side streams from the SEPoR process.

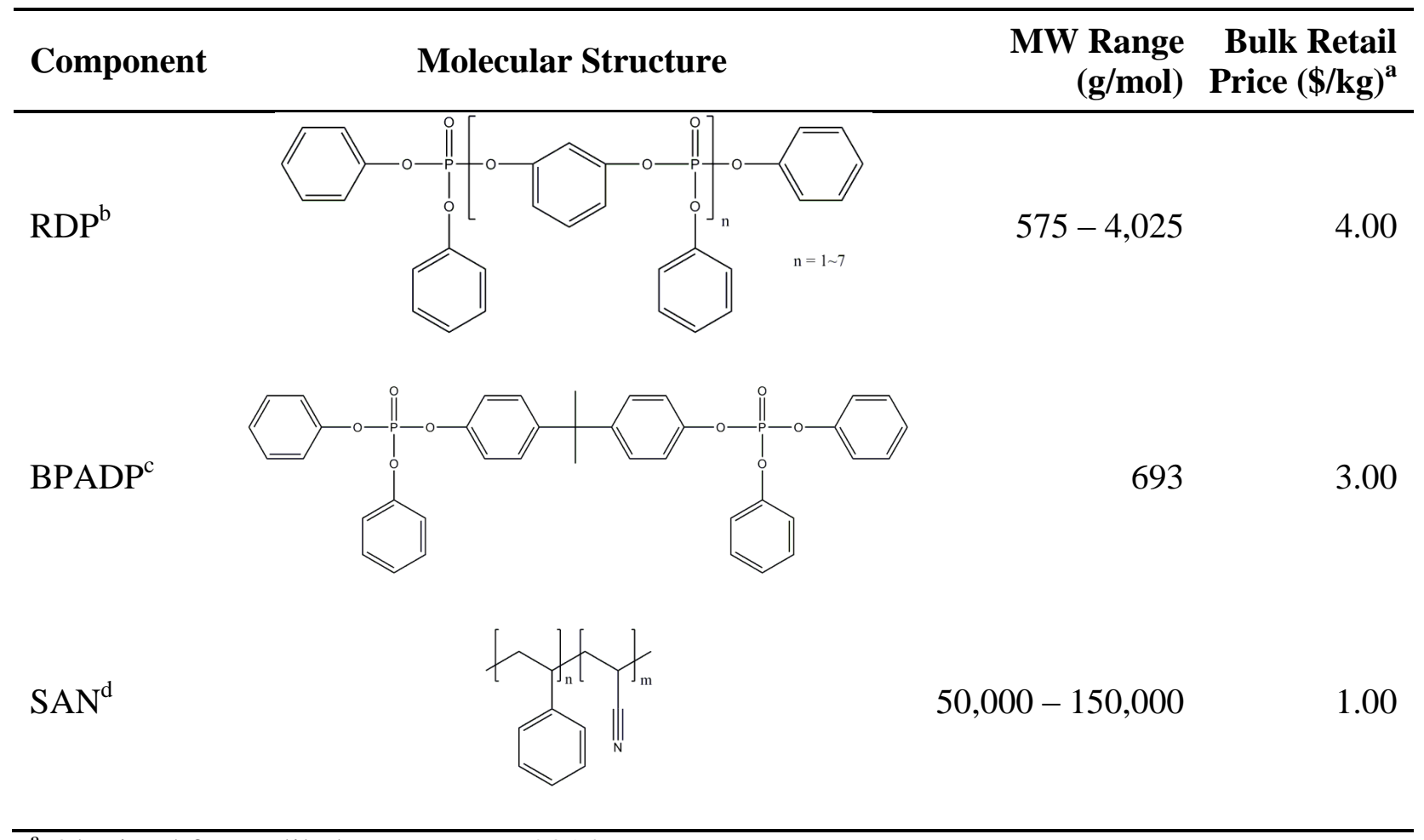

${ }^{\mathrm{a}}$ Obtained from alibaba.com, May 2014

${ }^{\mathrm{b}}$ Resorcinol bis-diphenylphosphate

${ }^{\mathrm{c}}$ Bisphenol A bis-diphenylphosphate

${ }^{\mathrm{d}}$ Poly(styrene-co-acrylonitrile) 
Table 2

Dimensionless variables and groups for ternary, SEC-SMB

\begin{tabular}{|c|c|c|c|}
\hline Symbol & Name & Definition & Description \\
\hline$\delta_{i}$ & $\begin{array}{l}\text { Retention } \\
\text { factor }\end{array}$ & $\delta_{i}=\varepsilon_{p} K_{s e, i}+\frac{D V}{\left(1-\varepsilon_{b}\right)}$ & $\begin{array}{l}\text { Measure of how much each } \\
\text { component is retained by the } \\
\text { sorbent }\end{array}$ \\
\hline$\alpha_{i}$ & Selectivity & $\alpha_{i}=\frac{\delta_{\mathrm{i}}}{\delta_{1}}$ & $\begin{array}{l}\text { Ratio of retention factors: } \\
\text { greater than one for } \\
\text { separation }\end{array}$ \\
\hline$\beta_{i}^{j}$ & - & $\beta_{i}^{j}=\ln \left(\frac{C_{\max , i}^{j}}{C_{\min , i}^{j}}\right)$ & $\begin{array}{l}\text { Natural log of ratio of max. } \\
\text { conc. to min. conc. of } \\
\text { standing component in zone } \\
\mathrm{j}\end{array}$ \\
\hline$\gamma_{i}$ & $\begin{array}{l}\text { Diffusivity } \\
\text { ratio }\end{array}$ & $\gamma_{i}=\frac{D_{p, \mathrm{i}}}{D_{p, 1}}$ & $\begin{array}{l}\text { Ratio of intraparticle } \\
\text { diffusivities }\end{array}$ \\
\hline$\lambda_{i}$ & $\begin{array}{l}\text { Size- } \\
\text { exclusion } \\
\text { ratio }\end{array}$ & $\lambda_{i}=\frac{K_{s e, \mathrm{i}}}{K_{s e, 1}}$ & $\begin{array}{l}\text { Ratio of size-exclusion } \\
\text { factors; equal to } \alpha \text { if there is } \\
\text { no dead volume }\end{array}$ \\
\hline$\phi$ & Phase ratio & $\phi=\frac{1-\varepsilon_{b}}{\varepsilon_{b}}$ & $\begin{array}{l}\text { Particle phase relative to bed } \\
\text { void }\end{array}$ \\
\hline$N_{D, i}$ & - & $N_{D, i}=\frac{\phi \varepsilon_{p} K_{s e, i} D_{p, i}}{R_{p}^{2}} \frac{L_{c}}{v}=\frac{t_{s}}{t_{D, i}}$ & $\begin{array}{l}\text { Step time relative to } \\
\text { diffusion time }\end{array}$ \\
\hline$N_{D}^{*}$ & - & $N_{D}^{*}=\frac{N_{D, 1}\left(\alpha_{2}-1\right)}{\phi \delta_{1}}=\frac{N_{D, i}\left(\alpha_{2}-1\right)}{\lambda_{i} \gamma_{i} \phi \delta_{1}}$ & $\begin{array}{l}\text { Component-independent } N_{D} \text {; } \\
N_{D, 1} \text { as base } \\
\text { Split } 1 \text { from } 2 \text { and } 3\end{array}$ \\
\hline & & $N_{D}^{*}=\frac{N_{D, 1}\left(\frac{\alpha_{3}}{\alpha_{2}}-1\right)}{\phi \delta_{2}}$ & Split 1 and 2 from 3 \\
\hline$N_{\Delta P}$ & - & $N_{\Delta P}=\frac{\Delta P_{\max } R_{p}^{2}}{37.5 \phi \mu L_{c} v}=\frac{t_{s}}{t_{c}^{I}}$ & $\begin{array}{l}\text { Step time relative to } \\
\text { pressure-limited convection } \\
\text { time }\end{array}$ \\
\hline$P_{e b}^{j}$ & $\begin{array}{l}\text { Peclet } \\
\text { number }\end{array}$ & $P_{e b}^{j}=\frac{v L_{c}}{E_{b}^{j}}=\frac{t_{D a x}}{t_{s}}$ & $\begin{array}{l}\text { Axial dispersion time } \\
\text { relative to step time }\end{array}$ \\
\hline$\Gamma^{j}$ & $\begin{array}{l}\text { Axial } \\
\text { dispersion } \\
\text { ratio }\end{array}$ & $\Gamma^{j}=\frac{E_{b}^{j}}{E_{b}^{I V}}$ & $\begin{array}{l}\text { Ratio of axial dispersion } \\
\text { coefficients of zone } \mathrm{j} \text { and } \\
\text { zone IV }\end{array}$ \\
\hline$P_{e b}^{*}$ & - & $\begin{array}{l}P_{e b}^{*}=\phi\left(\delta_{2}-\delta_{1}\right) P_{e b}^{j} \Gamma^{j} \\
P_{e b}^{*}=\phi\left(\delta_{3}-\delta_{2}\right) P_{e b}^{j} \Gamma^{j}\end{array}$ & $\begin{array}{l}\text { Zone-independent Peclet } \\
\text { number, Zone IV as base } \\
\text { Split } 1 \text { from } 2 \text { and } 3 \\
\text { Split } 1 \text { and } 2 \text { from } 3\end{array}$ \\
\hline
\end{tabular}


Table 3

SSWD equations for ternary SEC-SMB.

\begin{tabular}{|c|c|}
\hline Split 1 (solute 1 from solutes 2 and 3) & Split 2 (solutes 1 and 2 from solute 3 ) \\
\hline$u_{0}^{I}=v\left(1+\phi \delta_{3}+\frac{\beta_{3}^{I} \Gamma^{I} \phi\left(\delta_{2}-\delta_{1}\right)}{P_{e b}^{*} N^{I}}+\frac{\phi \beta_{3}^{I} \alpha_{3}^{2}\left(\delta_{2}-\delta_{1}\right)}{15 N_{D}^{*} \lambda_{3} \gamma_{3} N^{I}}\right)$ & (4a) $u_{0}^{I}=v\left(1+\phi \delta_{3}+\frac{\beta_{3}^{I} \Gamma^{I} \phi\left(\delta_{3}-\delta_{2}\right)}{P_{e b}^{*} N^{I}}+\frac{\phi \beta_{3}^{I} \alpha_{3}^{2}\left(\delta_{3}-\delta_{2}\right)}{15 \alpha_{2}^{2} N_{D}^{*} \lambda_{3} \gamma_{3} N^{I}}\right)$ \\
\hline$u_{0}^{I I}=v\left(1+\phi \delta_{1}+\frac{\beta_{1}^{I I} \Gamma^{I I} \phi\left(\delta_{2}-\delta_{1}\right)}{P_{e b}^{*} N^{I I}}+\frac{\phi \beta_{1}^{I I}\left(\delta_{2}-\delta_{1}\right)}{15 N_{D}^{*} N^{I I}}\right)$ & (4b) $u_{0}^{I I}=v\left(1+\phi \delta_{2}+\frac{\beta_{2}^{I I} \Gamma^{I I} \phi\left(\delta_{3}-\delta_{2}\right)}{P_{e b}^{*} N^{I I}}+\frac{\phi \beta_{2}^{I I}\left(\delta_{3}-\delta_{2}\right)}{15 N_{D}^{*} \lambda_{2} \gamma_{2} N^{I I}}\right)$ \\
\hline$u_{0}^{I I I}=v\left(1+\phi \delta_{2}-\frac{\beta_{2}^{I I I} \Gamma^{I I I} \phi\left(\delta_{2}-\delta_{1}\right)}{P_{e b}^{*} N^{I I I}}-\frac{\phi \beta_{2}^{I I I} \alpha_{2}{ }^{2}\left(\delta_{2}-\delta_{1}\right)}{15 N_{D}^{*} \lambda_{2} \gamma_{2} N^{I I I}}\right)$ & (4c) $u_{0}^{I I I}=v\left(1+\phi \delta_{3}-\frac{\beta_{3}^{I I I} \Gamma^{I I I} \phi\left(\delta_{3}-\delta_{2}\right)}{P_{e b^{N}}^{* I I}}-\frac{\phi \beta_{3}^{I I I} \alpha_{3}{ }^{2}\left(\delta_{3}-\delta_{2}\right)}{15 \alpha_{2}{ }^{2} N_{D}^{*} \lambda_{3} \gamma_{3} N^{I I I}}\right)$ \\
\hline$u_{0}^{I V}=v\left(1+\phi \delta_{1}-\frac{\beta_{1}^{I V} \phi\left(\delta_{2}-\delta_{1}\right)}{P_{e b}^{*} N^{I V}}-\frac{\phi \beta_{1}^{I V}\left(\delta_{2}-\delta_{1}\right)}{15 N_{D}^{*} N^{I V}}\right)$ & (4d) $\quad u_{0}^{I V}=v\left(1+\phi \delta_{1}-\frac{\beta_{1}^{I V} \phi\left(\delta_{3}-\delta_{2}\right)}{P_{e b}^{*} N^{I V}}-\frac{\phi \beta_{1}^{I V}\left(\delta_{3}-\delta_{2}\right)}{15 \alpha_{2}^{2} N_{D}^{*} N^{I V}}\right)$ \\
\hline$\frac{\Delta P}{L^{I}}=\frac{150 \mu u_{0}^{I} \phi^{2}}{4 R_{p}^{2}}$ & (6) \\
\hline$N_{\Delta P}=\frac{\Delta P_{\max } R_{p}^{2}}{37.5 \phi \mu L_{c} v}=\phi\left(1+\phi \delta_{3}+\frac{\beta_{3}^{I} \Gamma^{I} \phi\left(\delta_{2}-\delta_{1}\right)}{P_{e b}^{*} N^{I}}+\frac{\phi \beta_{3}^{I} \alpha_{3}^{2}\left(\delta_{2}-\delta_{1}\right)}{15 N_{D}^{*} \lambda_{3} \gamma_{3} N^{I}}\right)$ & $\left.\phi \delta_{3}+\frac{\beta_{3}^{I} \Gamma^{I} \phi\left(\delta_{3}-\delta_{2}\right)}{P_{e b}^{*} N^{I}}+\frac{\phi \beta_{3}^{I} \alpha_{3}{ }^{2}\left(\delta_{3}-\delta_{2}\right)}{15 \alpha_{2}{ }^{2} N_{D}^{*} \lambda_{3} \gamma_{3} N^{I}}\right)$ \\
\hline
\end{tabular}


Table 4

Summary of material properties, system parameters, and numerical parameters for VERSE simulations.

Column Packing Parameters

\begin{tabular}{|c|c|c|c|c|c|c|c|c|}
\hline \multirow{2}{*}{$\begin{array}{c}\boldsymbol{R}_{\boldsymbol{p}}(\boldsymbol{\mu m}) \\
\text { XAD-1180N }\end{array}$} & \multicolumn{2}{|c|}{$L_{c}(\mathbf{c m})$} & \multirow{2}{*}{\multicolumn{2}{|c|}{$I D(\mathbf{c m})$}} & \multirow{2}{*}{$\varepsilon_{b}$} & \multirow{2}{*}{$\varepsilon_{p}$} & \multirow{2}{*}{$\phi$} & \multirow{2}{*}{$D V(\% \mathrm{CV})$} \\
\hline & Batch & SMB & & & & & & \\
\hline 225 & 65 & 63 & \multicolumn{2}{|c|}{2.54} & 0.37 & 0.69 & 1.70 & 1.9 \\
\hline \multicolumn{9}{|c|}{ Mass Transfer Parameters } \\
\hline \multirow{2}{*}{ Component } & \multirow{2}{*}{$\begin{array}{c}D_{\infty}{ }^{\mathrm{e}} \\
\left(\mathrm{cm}^{2} / \mathrm{min}\right)\end{array}$} & $D_{p}$ & \multicolumn{2}{|c|}{$K_{s e}$} & \multicolumn{2}{|r|}{$\delta$} & \multirow{2}{*}{$\begin{array}{c}E_{b} \\
\left(\mathrm{~cm}^{2} / \mathrm{min}\right)\end{array}$} & \multirow{2}{*}{$\begin{array}{c}k_{f} \\
(\mathrm{~cm} / \mathrm{min})\end{array}$} \\
\hline & & $\left(\mathrm{cm}^{2} / \mathrm{min}\right)$ & Batch & SMB & Batch & SMB & & \\
\hline SAN & $1.0 \times 10^{-4}$ & $0.7 \times 10^{-5}$ & 0.63 & 0.61 & 0.43 & 0.42 & Chung & Wilson and \\
\hline BPADP & $1.0 \times 10^{-2}$ & $40.0 \times 10^{-5}$ & 1.00 & 0.96 & 0.69 & 0.66 & and Wen & Geankoplis \\
\hline RDP & $1.0 \times 10^{-3}$ & $10.0 \times 10^{-5}$ & 1.00 & 1.00 & 0.69 & 0.69 & correlation & correlation \\
\hline
\end{tabular}

Numerical Parameters

\begin{tabular}{cccccc}
\hline \multirow{2}{*}{ No. of axial elements } & \multicolumn{2}{c}{ Collocation Points } & & \multicolumn{2}{c}{ Tolerance } \\
\cline { 2 - 3 } \cline { 5 - 6 } & Axial & Particle & & Absolute & Relative \\
\hline 100 & 4 & 1 & & 0.001 & 0.001 \\
\hline${ }^{e}$ Bnnnnyyn
\end{tabular}

${ }^{\mathrm{e}}$ Brownian diffusivities (see Supplementary Materials, S.3). 
Table 5

Summary of designs for SMB runs.

\begin{tabular}{|c|c|c|c|c|c|c|c|c|c|c|}
\hline \multicolumn{2}{|c|}{ Design } & \multicolumn{8}{|c|}{ Flowrates (mL/min) } & \multirow[b]{2}{*}{$\begin{array}{c}\text { Switching } \\
\text { Time (min) }\end{array}$} \\
\hline Run & $\begin{array}{c}L_{c}^{f} \\
(\mathbf{c m})\end{array}$ & Feed & Desorbent & Extract & Raffinate & $\begin{array}{c}\text { Zone } \\
\text { I }\end{array}$ & $\begin{array}{c}\text { Zone } \\
\text { II }\end{array}$ & $\begin{array}{c}\text { Zone } \\
\text { III }\end{array}$ & $\begin{array}{c}\text { Zone } \\
\text { IV }\end{array}$ & \\
\hline 1 & 65 & 0.59 & 1.97 & 0.79 & 1.77 & 7.45 & 6.66 & 7.25 & 5.48 & 36.9 \\
\hline 2 & 65 & 0.4 & 1.18 & 0.49 & 1.09 & 4.59 & 4.1 & 4.5 & 3.41 & 59.7 \\
\hline 3 & 65 & 0.59 & 1.97 & 0.79 & 1.77 & 7.45 & 6.66 & 7.25 & 5.48 & 36.9 \\
\hline 4 & 63 & 0.36 & 0.54 & 0.39 & 0.51 & 2.21 & 1.82 & 2.18 & 1.67 & 119.6 \\
\hline 5 & 65 & 0.4 & 1.18 & 0.49 & 1.09 & 4.59 & 4.1 & 4.5 & 3.41 & 59.7 \\
\hline 6 & 63 & 0.36 & 0.65 & 0.45 & 0.56 & 2.59 & 2.14 & 2.5 & 1.94 & 102.5 \\
\hline
\end{tabular}

${ }^{\mathrm{f}} L_{c}$ is the packing length used in the SWD 
Table 6

Summary of results for SMB experiments.

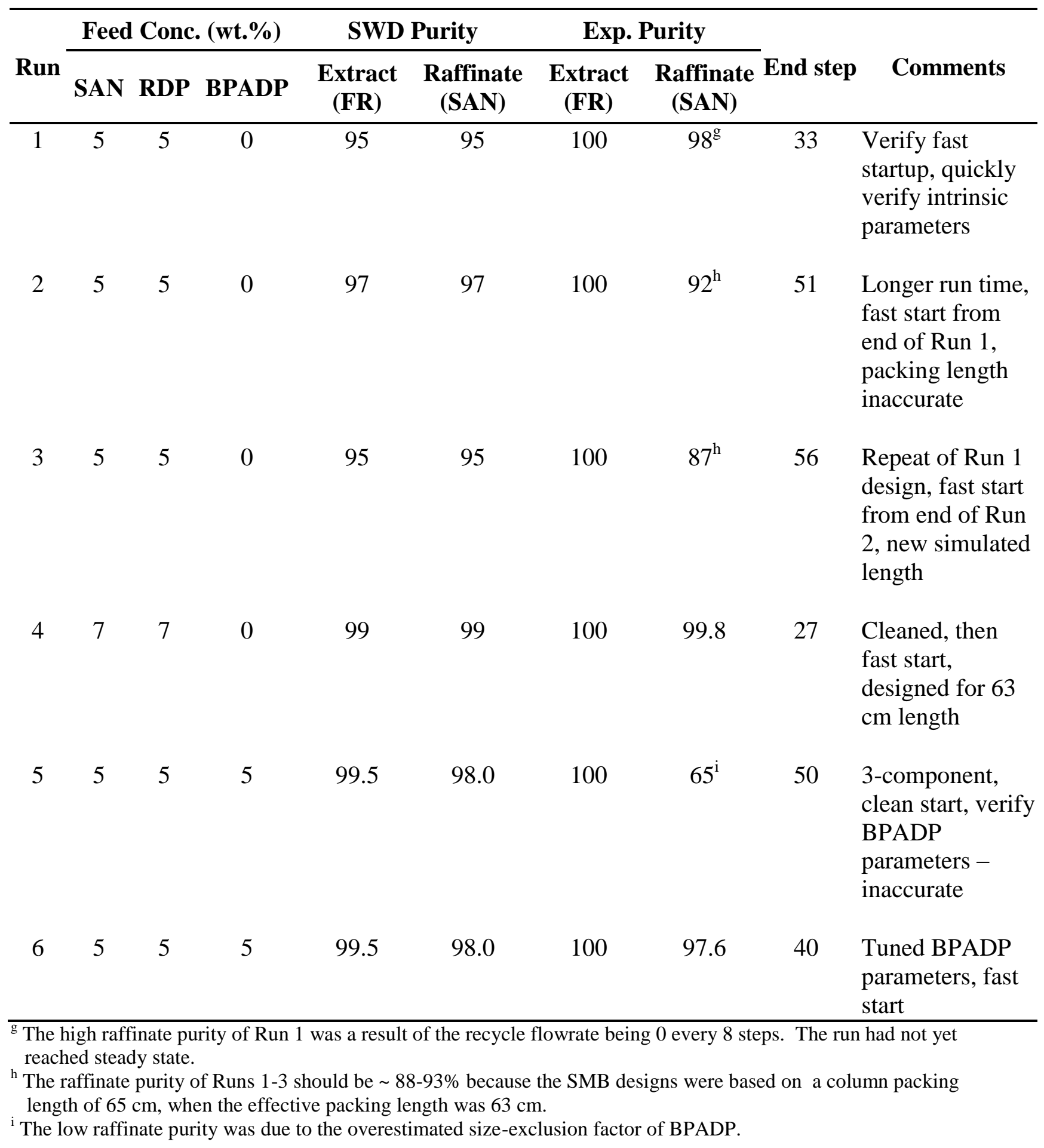


Table 7

Comparison of estimated costs ${ }^{\mathrm{j}}$ of SEC SMB and batch SEC for separating FRs from SAN.

\begin{tabular}{|c|c|c|}
\hline $\begin{array}{l}\text { Parameter } \\
\text { (10,000 tonnes FR/year) }\end{array}$ & Optimized SEC-SMB & Batch SEC \\
\hline \multicolumn{3}{|l|}{ Feed Concentration (wt.\%) } \\
\hline RDP & 5 & 5 \\
\hline BPADP & 5 & 5 \\
\hline SAN & 10 & 10 \\
\hline Product conc./Feed conc. & 0.83 & 0.10 \\
\hline Yield of each component & 99 & 99 \\
\hline Column configuration & $2-2-2-2$ & 100 units \\
\hline Column length (cm) & 55.2 & 126 \\
\hline Inner diameter (m) & 9.65 & 8.19 \\
\hline Feed flowrate (L/min) & 175 & 210 (per unit) \\
\hline Equipment cost (\$/kg FR) & 0.04 & 0.82 \\
\hline Solvent cost ( $\$ / \mathrm{kg}$ FR) & 0.18 & 6.05 \\
\hline Sorbent cost $(\$ / \mathrm{kg} F R)$ & 0.07 & 4.40 \\
\hline Total cost (\$/kg FR) & 0.29 & 11.27 \\
\hline
\end{tabular}

